\title{
Broadband acoustic quantification of stratified turbulence
}

\author{
Andone C. Lavery ${ }^{\mathrm{a})}$ and W. Rockwell Geyer \\ Woods Hole Oceanographic Institution, Department of Applied Ocean Physics and Engineering, Woods Hole, \\ Massachusetts 02543 \\ Malcolm E. Scully ${ }^{\text {b) }}$ \\ Center for Coastal Physical Oceanography, Old Dominion University, Norfolk, Virginia 23508
}

(Received 20 July 2012; revised 22 March 2013; accepted 7 May 2013)

\begin{abstract}
High-frequency broadband acoustic scattering techniques have enabled the remote, high-resolution imaging and quantification of highly salt-stratified turbulence in an estuary. Turbulent salinity spectra in the stratified shear layer have been measured acoustically and by in situ turbulence sensors. The acoustic frequencies used span $120-600 \mathrm{kHz}$, which, for the highly stratified and dynamic estuarine environment, correspond to wavenumbers in the viscous-convective subrange $\left(500-2500 \mathrm{~m}^{-1}\right)$. The acoustically measured spectral levels are in close agreement with spectral levels measured with closely co-located micro-conductivity probes. The acoustically measured spectral shapes allow discrimination between scattering dominated by turbulent salinity microstructure and suspended sediments or swim-bladdered fish, the two primary sources of scattering observed in the estuary in addition to turbulent salinity microstructure. The direct comparison of salinity spectra inferred acoustically and by the in situ turbulence sensors provides a test of both the acoustic scattering model and the quantitative skill of acoustical remote sensing of turbulence dissipation in a strongly sheared and salt-stratified estuary. (C) 2013 Acoustical Society of America. [http://dx.doi.org/10.1121/1.4807780]
\end{abstract}

PACS number(s): 43.30.Ft, 43.20.Fn, 43.30.Pc, 43.30.Zk [APL]

Pages: $40-54$

\section{INTRODUCTION}

Acoustic backscattering techniques provide a unique and powerful tool to rapidly and remotely investigate the physical properties of the ocean interior over a large range of important spatial and temporal scales. These techniques have been commonly used to image physical processes that occur in the ocean interior (Proni and Apel, 1975; Haury et al., 1979; Sandstrom et al., 1989; Trevorrow, 1998; Farmer and Armi, 1999), including, for example, internal waves (Orr et al., 2000; Moum et al., 2003), hydraulic jumps (Farmer and Smith, 1979; Cummins et al., 2006), bubbles (Medwin, 1977; Vagle and Famer, 1994), Langmuir circulation (Thorpe et al., 1994), suspended sediments (Young et al., 1982; Hay, 1983; Lynch et al., 1991; Thorne et al., 1991), and microstructure (Kraichnan, 1953; Thorpe and Brubaker, 1983; Seim et al., 1995; Seim, 1999; Ross and Lueck, 2003; Warren et al., 2003; Lavery et al., 2010a,b; Goodman and Sastre-Cordova, 2011). From an acoustics perspective, microstructure refers to fluctuations in temperature and salinity, resulting in fluctuations in sound speed and density (which in turn scatter sound), occurring at scales from sub-millimeter to tens of centimeters.

Over the past two decades, significant research effort has been directed at, not just imaging, but quantifying turbulent oceanic microstructure using high-frequency acoustic scattering techniques (Seim et al., 1995; Moum et al., 2003;

\footnotetext{
a) Author to whom correspondence should be addressed. Electronic mail: alavery@whoi.edu

${ }^{b}$ Current address: Woods Hole Oceanographic Institution, Department of Applied Ocean Physics and Engineering, Woods Hole, MA 02543.
}

Ross and Lueck, 2003; Warren et al., 2003; Goodman and Sastre-Cordova, 2011). These studies have employed a restricted number of high-frequency narrowband acoustic frequencies and achieved moderate success in obtaining agreement between predicted scattering levels and measured scattering levels. Inversions of the acoustic scattering data for microstructure parameters has been more challenging, in part because the problem is heavily under-determined, requiring many assumptions in order to perform inversions, and resulting in significant sources of error. One of the most egregious problems involves the inversion of scattering returns that are assumed to be due to turbulent microstructure, but are in fact due to one of the many other sources of scattering that have not been correctly classified. Depending on the environment, there can be either physical (e.g., microstructure, bubbles, and suspended sediments) or biological (including fish, squid, and zooplankton) source of scattering. Many of these scattering sources are patchy and intermittent across a broad range of spatial and temporal scales (Seuront et al., 2001) and can occur simultaneously, e.g. turbulent patches in which small zooplankton can act as passive tracers of the underlying turbulence (Ross et al., 2007). In situ measurements of the different sources of scatterers, obtained with instruments such as microstructure profilers, nets, and optical systems, can reduce the number of assumptions made in performing inversions, though the spatial and temporal sampling scales of these complementary measurements rarely match those of the acoustic measurements. Thus, though acoustic scattering techniques provide a rapid, highresolution, synoptic, remote-sensing alternative to more traditional sampling strategies, reducing the ambiguities in the quantitative interpretation of the acoustic returns, with the 
goal of accurate, remote classification and quantification of physical (and/or biological) scattering sources remains a key challenge.

This paper addresses some of these challenges by using broadband acoustic scattering techniques in the context of stratified turbulence, providing both high-resolution images as well as quantitative information about turbulent intensity. Emerging broadband acoustic scattering techniques (Foote et al., 2005; Lavery et al., 2010a,b; Stanton et al., 2010), can help with the accurate classification and quantification of stratified turbulence, as well as other scattering sources, on relevant spatial and temporal scales. Broadband acoustic scattering measurements result in acoustic spectra measured continuously over a range of frequencies and enable the use of pulse compression signal processing techniques to obtain high-resolution imaging capabilities (Chu and Stanton, 1998).

Broadband (160-600 kHz) acoustic scattering techniques have been successfully used previously by Lavery et al. (2010b) to quantify acoustic scattering from stratified turbulence generated by surface-trapped nonlinear internal waves during the Shallow Water 2006 Experiment (Tang et al., 2007). This environment was strongly temperature stratified, and salinity stratification played a minor role in determining the scattering. The broadband acoustic scattering measurements allowed high-resolution imaging and quantification of Kelvin-Helmholtz shear instabilities, which were not well resolved by the simultaneously measurements of temperature and velocity acquired with a profiling microstructure system. As a result of the acoustic frequencies used and the typical dissipation rates encountered, the acoustic measurements were almost entirely in the dissipation subrange. Acoustic inferences of relevant turbulent temperature microstructure parameters were in general agreement with the measurements performed with the profiling instruments, though it was not possible to match the sampling volumes. The in situ microstructure measurements using the profiling instrument provided very high-resolution measurements in the vertical, however the profiles were relatively sparse in time (one profile every few minutes depending on the depth) relative to the acoustic measurements (an acoustic "profile" of the entire water-column every second) or to the relevant scales of the physical processes being investigated.

In this paper, results of broadband $(120-600 \mathrm{kHz})$ acoustic scattering measurements of stratified turbulence in the Connecticut (CT) River estuary are presented, together with a suite of in situ measurements. This data set is unique because (1) the broadband acoustic scattering techniques allow the scattering as a function of frequency to be determined, thus providing a powerful tool for discriminating between sources of scattering and for quantifying stratified turbulence, (2) unlike intermittent samples collected with vertically profiling instruments, the in situ measurements in the CT River provide a temporally continuous record of mixing at multiple depths spanning the entire water column (though the vertical spacing of the sensors is too coarse to resolve the details of the structure that is evident in the acoustic returns), (3) the in situ and acoustic measurements were performed simultaneously over long time scales (in contrast to profiling instruments), (4) the highly saltstratified and energetic environment resulted in unprecedented scattering levels at short ranges, resulting in very high quality acoustic data, representing a "natural laboratory", and (5) a wide range of forcing conditions (stratification and mixing intensity) were measured through a tidal cycle.

The goals of this paper are to investigate the range of forcing parameters over which salinity microstructure dominates the scattering, and to show that when salinity microstructure dominates the scattering, as determined by the acoustic scattering spectra, (1) the direct comparison of salinity spectra measured acoustically and by in situ turbulence sensors are in close agreement, (2) the acoustic scattering model developed by Lavery et al. (2003) is valid in a different wavenumber regime than probed previously, also confirming the importance of salinity fluctuations in determining acoustic scattering, (3) the acoustic scattering is more sensitive to the dissipation rate of salinity variance than to the dissipation rate of turbulent kinetic energy, and (4) it is possible to infer values of dissipation rates of salinity variance that are consistent with inferences made from in situ measurements. These results illustrate that broadband acoustic scattering techniques, together with standard hydrographic measurements, can be a valuable tool for acoustical remote sensing of turbulent dissipation in a strongly sheared and salt-stratified estuary, complementing in situ measurements of turbulence.

The broadband acoustic measurements described here have also contributed to the overall understanding of mixing in highly stratified estuaries (Geyer et al., 2010) as a result of the high-resolution images afforded by the broadband techniques. Turbulent mixing in the presence of strong salinity stratification is one of the most important processes affecting the dynamics of estuaries (Scully et al., 2009). Turbulence intensities are three to four orders of magnitude higher than in the ocean and salinity stratification is also much greater than in oceanic environments due to the strong forcing by freshwater discharge. This combination leads to very small scales of turbulence, requiring small sampling volumes and high sampling frequencies to resolve the smallest scales. Further complicating the problem, turbulence is produced by both shear layers and boundary layers in strongly stratified estuaries, and over a single tidal cycle there is large variability in both the stratification and forcing. Geyer et al. (2008) have developed a suite of in situ instruments, including the mobile array for sensing turbulence (MAST), to address some of these challenges. However, the broadband acoustic scattering measurement have resulted in images (Geyer et al., 2010) of stratified turbulence at unprecedented resolution (Fig. 1) and have revealed a new mixing regime associated to shear instability at high Reynolds number $(\operatorname{Re}>500000)$. Though the imaging capabilities of broadband acoustics were instrumental in the interpretation of these high-Re number shear instabilities, it is the synergy between the acoustics and in situ data that has provided unique observations of the structure and evolution of stratified turbulence at high-Reynolds number and provided the opportunity to quantify the structure of stratified turbulence. 

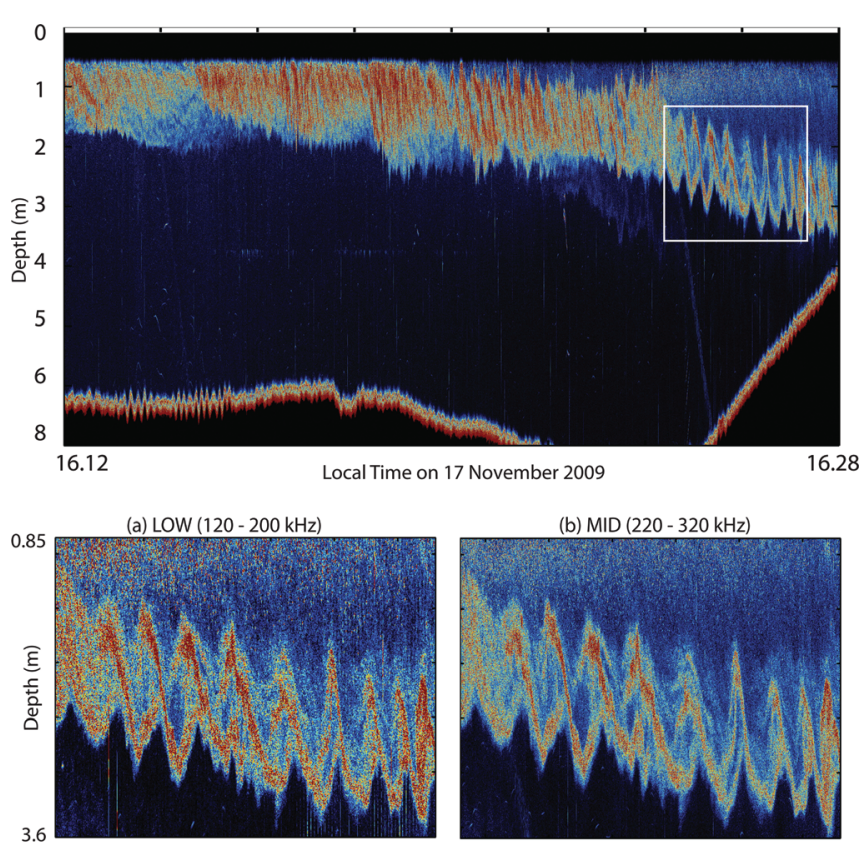

(c) $\mathrm{HL}(350-470 \mathrm{kHz})$

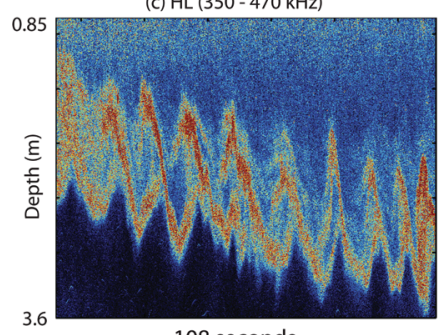

108 seconds

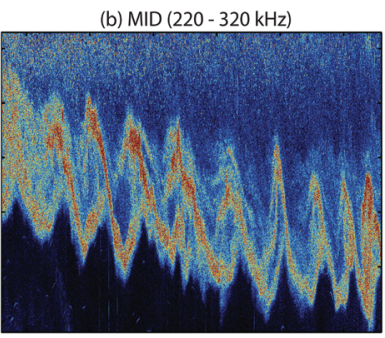

(d) $\mathrm{HH}(450-600 \mathrm{kHz})$

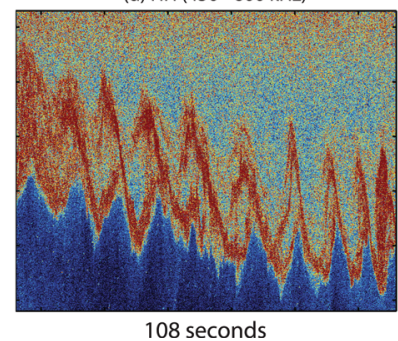

FIG. 1. Broadband acoustic backscattering along a transect at the mouth of the CT River on 17 November 2009. The image shows the envelope of the compressed pulse output, $E_{\mathrm{CP}}$, in linear space for the MID frequency channel, spanning 220-320 kHz. Shear instabilities are apparent. The insets (a)-(d) show $E_{\mathrm{CP}}$ for the four different broadband frequencies, illustrating frequency dependent scattering features.

Co-located measurements of acoustic backscatter and turbulence have provided far greater spatial-temporal resolution of turbulent structures than could have been accomplished with turbulence sensors alone. In this paper, the broadband measurements allow the dissipation rate of salinity variance associated to shear instabilities to be imaged at higher resolution than has been possible by previous techniques.

\section{STRATIFIED TURBULENCE AND ACOUSTIC SCATTERING FROM TURBULENT MICROSTRUCTURE}

In this section, mathematical models for turbulence in highly sheared and stratified environments are presented, followed by the associated models for acoustic scattering from homogeneous and isotropic turbulent microstructure, and the approach taken to invert acoustic scattering for turbulence parameters. Based on the classical homogeneous and isotropic turbulence model (Bachelor, 1959; Tennekes and Lumley, 1972; Dillon and Caldwell, 1980), the wavenumber spectra of temperature (T) and salinity $(\mathrm{S})$ are separated into three broad wavenumber regimes: the inertial-convective subrange, viscous-convective subrange, and viscous-diffusive subrange. The acoustic measurements that will be presented in later sections fall entirely in the viscous-convective subrange for salinity (Fig. 2), while the in situ measurements span both the inertial-convective and viscous-convective subranges.

\section{A. Tand $S$ spectra in the inertial-convective subrange}

The 1D wavenumber spectra for $T, S$, and their cospectrum, in the inertial-convective subrange, are given by

$$
\begin{aligned}
& \phi_{T, S}^{i c}(k)=C^{*} \chi_{T, S} \epsilon^{-1 / 3} k^{-5 / 3} \quad \text { and } \\
& \phi_{T-S}^{i c}(k)=C^{*}\left(\chi_{T} \chi_{S}\right)^{1 / 2} \epsilon^{-1 / 3} k^{-5 / 3}, \quad k_{b}<k<k^{*},
\end{aligned}
$$

where $k_{b}$ is the buoyancy wavenumber, given by $k_{b}=\left(N^{3} /\right.$ $\varepsilon)^{\frac{1}{2}}$, where $N=\sqrt{-(g / \rho)(d \rho / d z)}$ is the buoyancy frequency, $\rho$ is density and $g=9.8 \mathrm{~m} / \mathrm{s}^{2}$ is the acceleration due to gravity, and $k^{*}=0.036 k_{\nu}$, where $k_{\nu}=\left(\varepsilon / \nu^{3}\right)^{1 / 4}$ is the Kolmogorov wavenumber, $v\left(\approx 1.3 \times 10^{-6} \mathrm{~m}^{2} / \mathrm{s}\right)$ is the molecular viscosity, and $\varepsilon$ is the dissipation rate of turbulent kinetic energy $\left(\mathrm{m}^{2} / \mathrm{s}^{3}\right)$. The dissipation rates of temperature

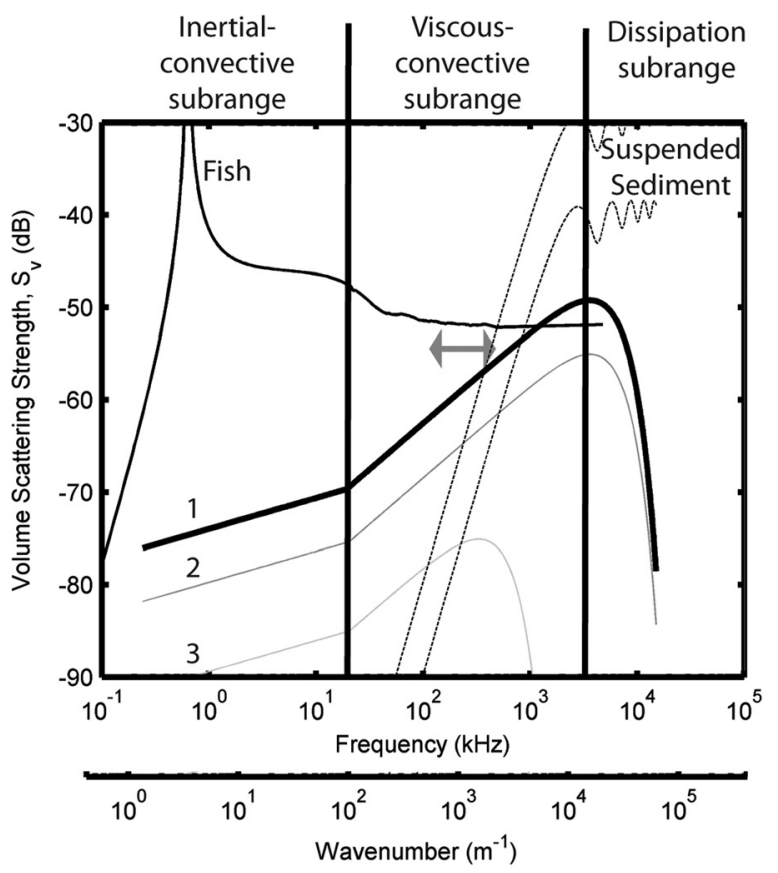

FIG. 2. Predicted volume scattering strength $(\mathrm{dB})$ for the dominant scattering sources observed in the CT River: Suspended sediments (dashed lines), using the mean measured fine sand grain size of $100 \mu \mathrm{m}$ and two different concentrations that give scattering levels in the range of the actual observations; fish, based on a spherical air-filled swim-bladder of $1 \mathrm{~cm}$ diameter; and predicted scattering due to microstructure (curves labeled 1-3). Curve 1 (black line) includes all contributions to scattering from density and sound speed, curve 2 (gray line) includes the contribution to scattering from sound speed only (including both temperature and salinity components), curve 3 (light gray line) includes the contribution to scattering from the temperature component of sound speed alone. The microstructure model parameters were taken from the mean values observed during the mid-ebb during the anchor station on 18 November 2009. The gray arrow indicates the frequency range of the broadband acoustic backscattering system. Unlike the in situ measurements, which span the inertial and viscous-convective subranges, the acoustic measurements are completely within the viscousconvective subrange. It can be seen that the contribution to scattering from temperature fluctuations is $20 \mathrm{~dB}$ smaller than the contribution from salinity fluctuations, however, the dissipation roll off for temperature occurs in the band of acoustic wavenumbers used in this study. 
and salinity variance are given by $\chi_{T}\left({ }^{\circ} \mathrm{C}^{2} / \mathrm{s}\right)$ and $\chi_{S}\left(\mathrm{psu}^{2} / \mathrm{s}\right)$, respectively. $C^{*}$ is a dimensionless constant, estimated to be given by $C^{*}=0.4$ (Sreenivasan, 1995), though values as high as 0.955 have been reported (Dillon and Caldwell, 1980). An upper-bound model based on the $1 \mathrm{D}$ wavenumber spectra has been used for the T-S co-spectrum $\left(\phi_{T-S}\right)$, which assumes perfect correlation between temperature and salinity (Washburn et al., 1996). Ross et al. (2004) have suggested that the T-S co-spectrum term should be given by an upperbound model that assumes perfect correlation between temperature and salinity based on the 3D isotropic wave number spectra instead of an upper-bound model based on the 1D wave number spectra, as suggested by Washburn et al. (1996). However, it can be shown that the two are equivalent for 1D wave number spectra with a power-law dependence $\left(\phi_{T, S} \propto k^{N^{\alpha}}\right.$ for any $\left.N^{\alpha}\right)$, as would be expected in the inertialconvective and viscous-convective subranges.

\section{B. T and S spectra in the viscous-convective subrange}

There is a viscous-convective subrange for wavenumbers between $k^{*}$ and the Batchelor wavenumber, $k_{B T, S}$ $=\left(\varepsilon /\left(\nu D_{T, S}^{2}\right)\right)^{1 / 4}$, where $D_{T} \approx 1.5 \times 10^{-7}\left(\mathrm{~m}^{2} / \mathrm{s}\right)$ and $D_{S} \approx$ $1.5 \times 10^{-9}\left(\mathrm{~m}^{2} / \mathrm{s}\right)$ are the molecular diffusivities of temperature and salt, respectively. The viscous-convective subrange in the highly salt-stratified Connecticut River estuary extends many decades due to the high values of $\varepsilon$ (Fig. 2). In this subrange, the 1D wavenumber spectra for $\mathrm{T}, \mathrm{S}$, and their co-spectrum, are given by

$$
\begin{aligned}
& \phi_{T, S}^{v c}(k)=q \chi_{T, S}\left(\frac{\varepsilon}{\nu}\right)^{-1 / 2} k^{-1} \text { and } \\
& \phi_{T-S}^{v c}(k)=q\left(\chi_{T} \chi_{S}\right)^{1 / 2}\left(\frac{\varepsilon}{\nu}\right)^{-1 / 2} k^{-1},
\end{aligned}
$$

where $q$ is a dimensionless constant estimated to be given by $q=3.7$ (Oakey, 1982). $k^{*} / k_{\nu}=(C / q)^{3 / 2}$ is chosen such that the $1 \mathrm{D}$ wavenumber spectra in the inertial- and viscoussubranges match at $k^{*}$. An upper-bound model based on the 1D wavenumber spectra has again been used for $\phi_{T-S}$.

\section{Acoustic scattering from turbulent microstructure}

The scattering cross-section per unit volume, with units of inverse length, for homogeneous and isotropic fluctuations in temperature and salinity, is given by [Eq. (23) in Lavery et al., 2003]

$$
\begin{aligned}
\sigma_{V}(k) & =\sigma_{V}^{T}(k)+\sigma_{V}^{S}(k)+\sigma_{V}^{T-S}(k) \\
& =-\frac{k^{4}}{K}\left(A^{2} \frac{\mathrm{d} \phi_{T}(K)}{d k}+B^{2} \frac{\mathrm{d} \phi_{S}(K)}{d k}+2 A B \frac{\mathrm{d} \phi_{T-S}(K)}{d k}\right),
\end{aligned}
$$

where $k$ is the incident acoustic wave number $(k=2 \pi / \lambda$, where $\lambda$ is the acoustic wave length), $K$ is the Bragg wave number ( $K=2 k$ in the backscattering direction), and $\phi_{T}(K)$, $\phi_{S}(K)$, and $\phi_{T-S}(K)$ are the wave number spectra for temperature fluctuations, salinity fluctuations, and the correlation between temperature and salinity fluctuations, all evaluated at the Bragg wave number. $A$ and $B$ contain terms that represent the fractional change in sound speed and density due to temperature and salinity changes, respectively. In the backscattering direction $A=a-\alpha$ and $B=b+\beta$, where $a=\left(1 / c_{w}\right)\left(\partial c_{w} / \partial T\right), b=\left(1 / c_{w}\right)\left(\partial c_{w} / \partial S\right), \alpha=-(1 / \rho)(\partial \rho /$ $\partial T)$, and $\beta=(1 / \rho)(\partial \rho / \partial S)$, where $c_{w}$ is the sound speed. The volume backscattering strength, $S_{V}=10 \log _{10} \sigma_{V}$, with units of decibels relative to $1 \mu \mathrm{Pa}$ at $1 \mathrm{~m}$, is a function of the acoustic frequency (or wavenumber) and is referred to as a scattering spectrum. Substituting Eq. (1) into Eq. (3), $\sigma_{V}$ in the inertial-convective subrange is given by

$$
\begin{aligned}
\sigma_{V}^{i c}(k)= & \frac{5}{3} 2^{-11 / 3}\left(A^{2} \chi_{T}+B^{2} \chi_{S}\right. \\
& \left.+2 A B\left(\chi_{T} \chi_{S}\right)^{1 / 2}\right) C^{*} \epsilon^{-1 / 3} k^{1 / 3}
\end{aligned}
$$

$\chi_{T}$ can be eliminated from Eq. (4) using $\chi_{T}=\chi_{S}(\partial \bar{T} / \partial \bar{S})^{2}$. Thus

$$
\begin{aligned}
\sigma_{V}^{i c}(k) & =\frac{5}{3} 2^{-11 / 3} \Psi^{a c} C^{*} \chi_{S} \epsilon^{-1 / 3} k^{1 / 3} \\
& =\frac{5}{3} 2^{-11 / 3} \Psi^{a c} \phi_{S}^{i c}(k) k^{2}=\frac{5}{3} 2^{-11 / 3} \Psi^{a c} S^{i c}(k),
\end{aligned}
$$

where $\Psi^{a c}=A^{2}(\partial \bar{T} / \partial \bar{S})^{2}+B^{2}+2 A B(\partial \bar{T} / \partial \bar{S})$, and $S^{i c}(k)$ is the salinity gradient spectrum in the inertial-convective subrange.

Substituting Eq. (2) into Eq. (3), $\sigma_{V}$ in the viscousconvective surbrange is given by

$$
\begin{aligned}
\sigma_{V}^{v c}(k) & =2^{-3} \Psi^{a c} q \chi_{S}\left(\frac{\varepsilon}{\nu}\right)^{-1 / 2} k \\
& =2^{-3} \Psi^{a c} \phi_{S}^{v c}(k) k^{2}=2^{-3} \Psi^{a c} S^{\nu c}(k),
\end{aligned}
$$

where $S^{\nu c}(k)$ is the salinity gradient spectrum in the viscousconvective subrange.

\section{Inversion of acoustic spectra for dissipation parameters}

To invert measurements of acoustic scattering for the parameters $\chi_{S}$ and $\varepsilon$, or even to predict acoustic scattering from turbulent microstructure, it is necessary to first estimate the parameters that make up $\Psi^{a c}$, namely, $A, B$, and $\partial \bar{T} / \partial \bar{S}$. These parameters can be deduced from conductivity, temperature, and depth (CTD) measurements, not requiring specialized microstructure instrumentation, and do not vary significantly compared to the variations in $\chi_{S}$ and $\varepsilon$ (this will be illustrated for the CT River data in later sections). However, an additional complication in inverting the acoustic scattering measurements for $\chi_{S}$ and $\varepsilon$ arises from the fact that the acoustic scattering results from a combination of these two parameters. Specifically, the acoustic scattering is proportional to $\chi_{S} \varepsilon^{-1 / 3}$ in the inertialconvective subrange and to $\chi_{S} \varepsilon^{-1 / 2}$ in the viscousconvective subrange. To infer $\chi_{S}$ and $\varepsilon$ individually from the 
scattering measurements, either assumptions are needed about the turbulent mechanisms linking $\chi_{S}$ and $\varepsilon$ (for example, by assuming a mixing efficiency) or additional information on one of these parameters must be used from alternative measurements. Assuming a balance between the production and dissipation of salinity variance (ignoring the effects of temperature on density), then $\varepsilon$ can be related to $\chi_{S}$ through a flux Richardson number, $R_{f}$, the ratio of vertical buoyancy flux to shear production of turbulent kinetic energy (or mixing efficiency), and typical mean salinity gradients, $\partial \bar{S} / \partial z$, by, $R_{f}=g \beta \chi_{S} /[2 \epsilon(\partial \bar{S} / \partial z)]$.

Substituting this expression for the flux Richardson number into Eq. (6) allows $\chi_{S}$ and $\varepsilon$ to be expressed in terms of $R_{f}$ and the spectral levels measured acoustically in the viscous-convective subrange, given by

$$
\begin{aligned}
& \chi_{S}=\left(\frac{\sigma_{V}^{\nu c}}{2^{-3} \Psi^{a c} q k}\right)^{2}\left(\frac{g \beta}{2 R_{f}}\right)\left(\frac{\partial \bar{S}}{\partial z}\right)^{-1} \nu^{-1} \text { and } \\
& \varepsilon=\left(\frac{\sigma_{V}^{\nu c}}{2^{-3} \Psi^{a c} q k}\right)^{2}\left(\frac{g \beta}{2 R_{f}}\right)^{2}\left(\frac{\partial \bar{S}}{\partial z}\right)^{-2} \nu^{-1} .
\end{aligned}
$$

Similar expressions can be derived for $\chi_{S}$ and $\varepsilon$ in the inertial-convective subrange, though the acoustic scattering measurements in this paper fall entirely into the viscousconvective subrange of salinity microstructure, which dominates the scattering from microstructure in the CT River estuary. It is important to note that in the viscous-convective subrange, the estimate of $\chi_{s}$ depends on the 1st power of $R_{f}$ and $\partial \bar{S} / \partial z$, whereas the estimate of $\varepsilon$ depends on $R_{f}^{2}$ and $(\partial \bar{S} / \partial z)^{2}$, so $\varepsilon$ is considerably more sensitive to the assumed value of mixing efficiency and estimates of mean salinity gradients.

\section{METHODS AND MEASUREMENTS}

A suite of simultaneous acoustic and in situ turbulence measurements were performed, capitalizing on a highfrequency broadband acoustic scattering system (Lavery et al., 2010a) and the mobile array for sensing turbulence (MAST) (Geyer et al., 2008). Both systems recorded GPS time and location in order to synchronize the measurements. During the majority of the measurements, the acoustic system was mounted on the opposite side of the vessel from the MAST (lateral displacement approximately $6 \mathrm{~m}$ ), causing some phase shifts and de-correlation between the remote acoustic and in situ measurements. However, similar wave structures were observed with both sensors (Geyer et al., 2010), though the lateral correlation scale of turbulent parameters associated to shear instabilities is an open research question.

\section{A. Field site}

The measurements described here were conducted in the Connecticut (CT) River estuary (Fig. 3) from 16-20 November 2009 on board the RV Tioga. The Connecticut is a highly stratified, salt-wedge type estuary with tidal currents of approximately $1 \mathrm{~m} / \mathrm{s}$. All measurements were conducted

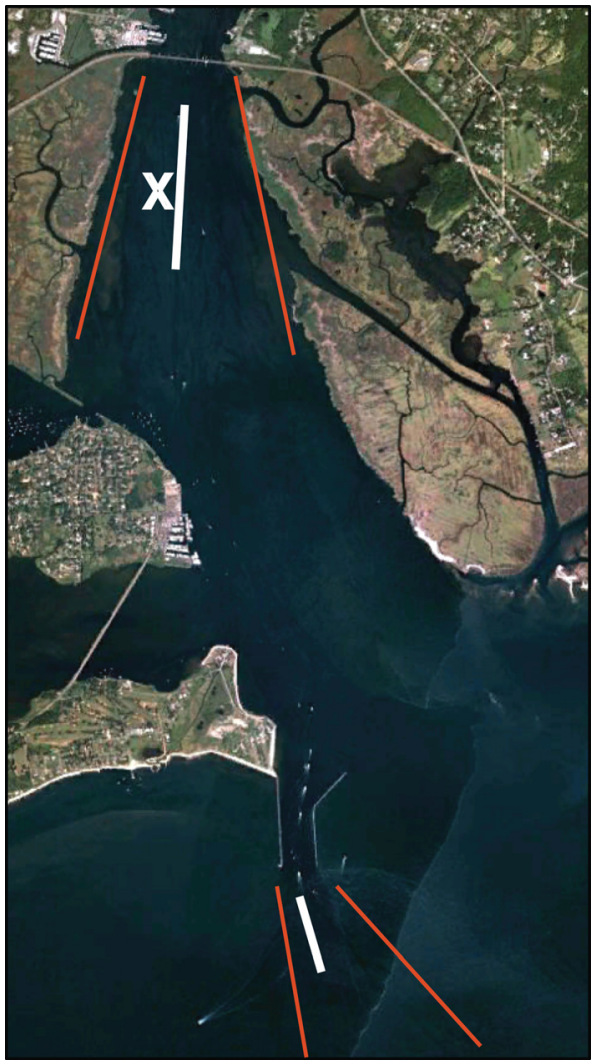

FIG. 3. (Color online) Google Earth image of the lower CT River estuary. The white lines illustrate transects performed at the two locations just downriver of major constrictions (illustrated by red lines). The white star shows the location of the anchor station performed on 18 November 2009. The width of the river at the bridge at the north end of the map is $0.5 \mathrm{~km}$.

during ebb tides with the vessel slowly steaming upstream against the ebbing current or at a fixed anchor station. Measurements were performed at two locations downstream of major constrictions which are locations typically characterized by strong horizontal density gradients due to the channel expansion. Multiple along-river transects were performed at both these locations during each ebb tide. The transects were approximately $500 \mathrm{~m}-1 \mathrm{~km}$ long, and the water depth was typically between 6-10 m. During one ebb tide, measurements were made at a stationary anchor station just downstream (approximately $300 \mathrm{~m}$ ) of one of the major constriction. River discharge throughout the measurement time period was moderate $\left(600-800 \mathrm{~m}^{3} / \mathrm{s}\right)$, yielding highly salt-stratified conditions.

The acoustic, hydrographic, and in situ turbulence measurements show that the early, middle and late phases of the ebb have very distinct characteristics (Figs. 4-6). During the early ebb [Fig. 4(a)], the pycnocline was in the upper part of the water column, with strong outflow of nearly fresh water at the surface and a strong shear zone across the pycnocline [Figs. 5(a) and 6]. Shear instabilities with amplitudes typically smaller than $1 \mathrm{~m}$ were observed in the upper part of the water column. The near-bottom velocity was weak, and bottom stress had no influence on the turbulence within the pycnocline. During the middle ebb [Fig. 4(b)], the pycnocline and velocity shear broadened to extend through the water column. Near-bottom velocity was still weak [Fig. 6(b)]. 
(a) Early Ebb

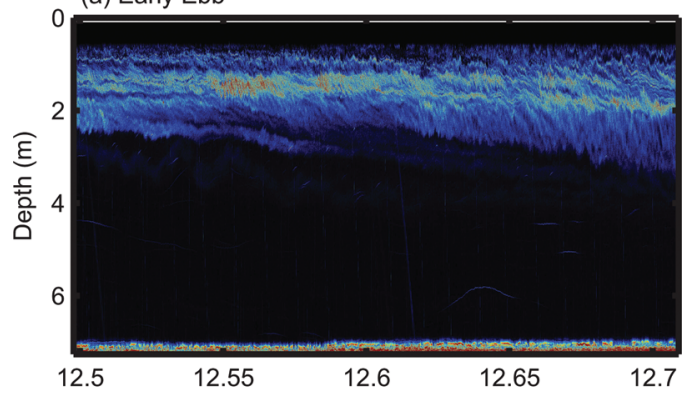

(b) Midd Ebb

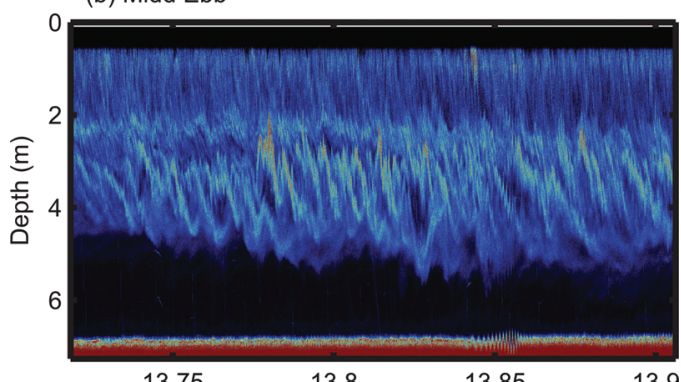

(c) Late Ebb

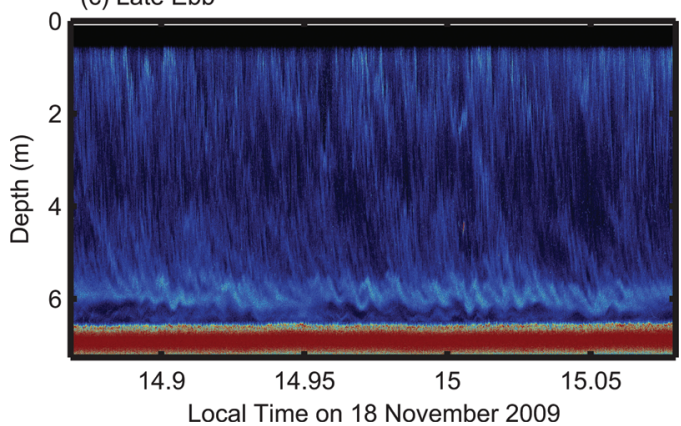

FIG. 4. Examples of broadband acoustic backscattering, $E_{\mathrm{CP}}$ for the MID frequency channel, at different times during the ebb tide at the anchor station on 18 November 2009: (a) Early ebb, characterized by strong outflow of nearly fresh water at the surface, (b) mid-ebb, characterized by welldeveloped stratified turbulence and shear instabilities, and (c) late ebb, characterized by significant lower stratification.

Larger amplitude (1-3 m) shear instabilities were frequently observed mid-water-column, with 10-20 m wavelengths. During the late ebb [Fig. 4(c)], salt was entirely flushed from this portion of the estuary, shear instabilities were rarely observed, near-bottom currents increased markedly, and boundary-layer turbulence dominated through the water column, with a corresponding increase in suspended sediment load [Figs. 4(c), 5(c), and 6].

\section{B. In situ turbulence measurements}

The MAST consists of a 10-m long, rigid instrument package, with eight adjustable instrument brackets, that is suspended from a research vessel (Geyer et al., 2008). Colocated, in situ measurements of velocity, conductivity, and temperature are obtained by the following sensors on each instrument bracket: (1) a Sontek acoustic Doppler velocimeter (ADV) (25 Hz sampling rate); (2) a Seabird Electronics SBE-7 micro-conductivity probe $(300 \mathrm{~Hz}$ sampling rate); (a) Salinity

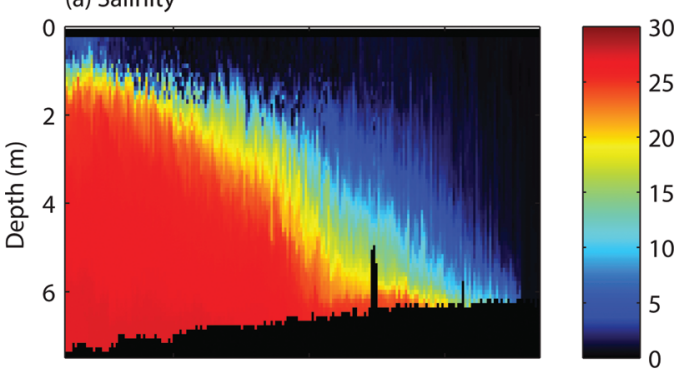

(b) Temperature

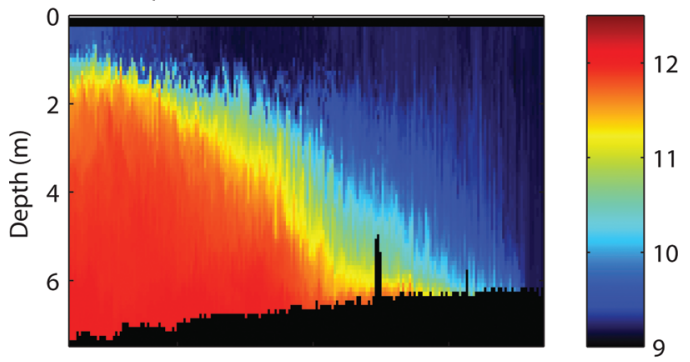

(c) OBS (Relative Units)

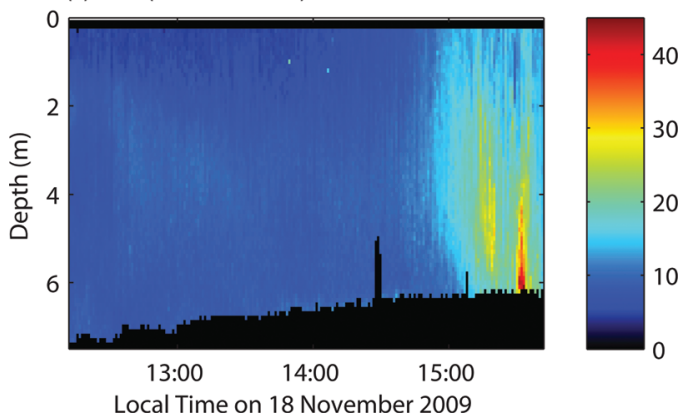

FIG. 5. (a) Salinity, (b) temperature, and (c) optical backscatter (OBS) during the ebb tide at the anchor station on 18 November 2009 from continuous CTD profiles. Increases in optical backscatter during the late ebb, due to increased levels of boundary layer turbulence and re-suspended sediment, are apparent.

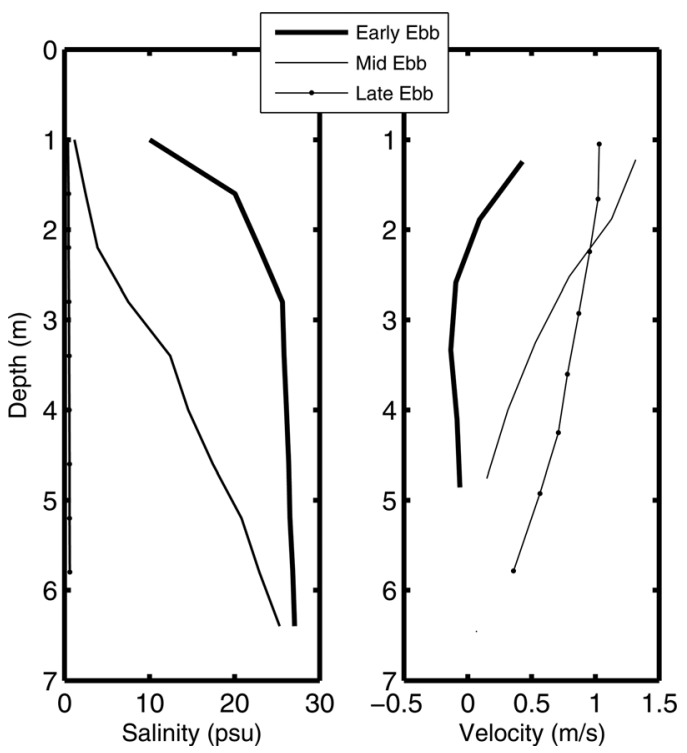

FIG. 6. (a) Salinity and (b) velocity profiles as a function of depth at three different times during the ebb tide at the anchor station on 18 November 2009 obtained from the CTD and MAST measurements. 
and (3) a conductivity, pressure and depth (CTD) sensor (Manufacturer RBR: $6 \mathrm{~Hz}$ sampling rate). The depth of the sensors depends on the angle of the MAST in the water, which in turn depends on the water depth. The depth of the MAST was continuously adjusted throughout the measurements. In contrast to measurements performed by other techniques, the MAST provides continuous measurements of turbulence quantities at multiple depths, allowing detailed comparison between the structures revealed by the acoustic backscattering and the continuous turbulence measurements.

\section{In situ turbulence spectra}

Data from the MAST were used to infer $\varepsilon$ and $\chi_{S}$ at each of the eight instrument brackets. Spectral quantities were calculated over $30 \mathrm{~s}$ intervals in order to maximize the tradeoff between temporal resolution and spectral uncertainty. Estimates of $\varepsilon$ were obtained from the height of the inertial subrange of the vertical velocity fluctuations measured by the ADVs (Grant et al., 1984; Shaw et al., 2001). Frequency spectra were converted to wavenumber spectra using the frozen turbulence hypothesis (Tennekes and Lumley, 1972) in conjunction with the horizontal advection speed measured by the ADVs. The inertial subrange was assumed to occur at wavenumbers larger than the Ozmidov scale (i.e., $k>k_{b}$ ). Because estimates of the Ozmidov scale require knowledge of $\varepsilon$, an iterative procedure was used to identify the inertial subrange. To ensure that spectra had a region with a clear $-5 / 3$ slope, only portions of the inertial subrange above the ADV noise floor were used. Under energetic conditions, a clear $-5 / 3$ inertial subrange was evident. However, there were some periods when turbulent energy was low and density stratification was strong, when no inertial subrange could be resolved by the ADVs. These data were excluded from the analysis.

Estimates of $\chi_{S}$ were obtained from the conductivity spectra measured by the micro-conductivity sensors. Using the independent estimates of $\varepsilon$, we fit the observed conductivity spectra to a model that contains both the inertialconvective and viscous-convective subrange. Following Washburn et al. (1996) and Nash and Moum (1999, 2002), assuming $\chi_{T}=\chi_{S}(\partial \bar{T} / \partial \bar{S})^{2}$, the relationship between conductivity and T-S can be linearized so that the conductivity spectra can be directly related to the salinity spectra (and hence $\chi_{S}$ ) as

$$
\begin{aligned}
\phi_{C}(k) & =\phi_{S}(k)\left[a_{C}^{2}\left(\frac{\partial \bar{T}}{\partial \bar{S}}\right)^{2}+b_{C}^{2}+2 a_{C} b_{C}\left(\frac{\partial \bar{T}}{\partial \bar{S}}\right)\right] \\
& =\Psi^{\mathrm{MAST}} \phi_{S}(k),
\end{aligned}
$$

where $a_{C}=\partial C / \partial T, \quad b_{C}=\partial C / \partial S, \quad$ and $\quad \Psi^{\mathrm{MAST}}$ $=a_{C}^{2}(\partial \bar{T} / \partial \bar{S})^{2}+b_{C}^{2}+2 a_{C} b_{C}(\partial \bar{T} / \partial \bar{S})$, in analogy to $\Psi^{a c}$. In the CT River, salinity gradients dominate over temperature, so the contribution of temperature [1st term in Eq. (8)] and the T-S co-spectrum [last term in Eq. (8)] can be safely ignored. Without significant contribution from temperature, the conductivity spectra have well-resolved inertial-convective and viscous-convective subranges (Fig. 7). We assume

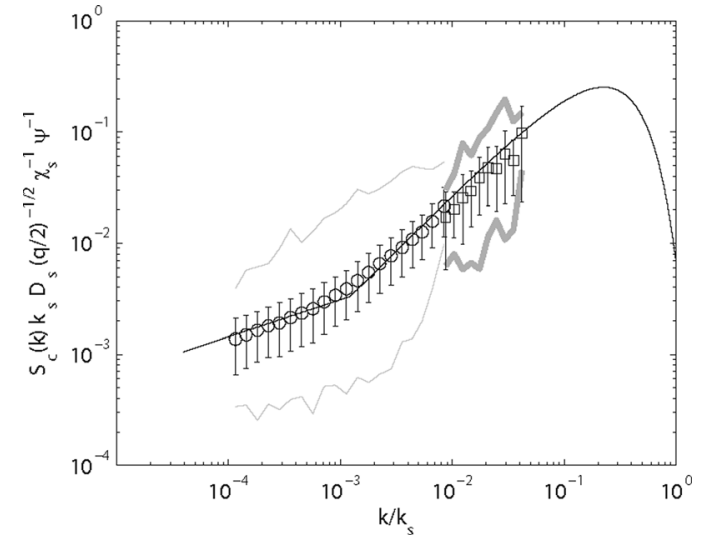

FIG. 7. Comparison of non-dimensional conductivity spectra from the MAST with similarly non-dimensionalized acoustic spectra during the midebb, using the same normalization as in Nash and Moum (2002; Fig. 8). The gray lines indicate the maximum and minimum range of the measured spectra. Circles (conductivity) and squares (acoustics) represent bin-averaged values and vertical lines represent 1 standard deviation. The smooth black line shows the theoretical Batchelor spectrum including the inertialconvective, viscous-convective, and dissipation subranges.

the inertial-convective subrange begins at wavenumbers where $k>k_{b}$ and transitions to the viscous-convective subrange where $k=k^{*}$. The viscous-convective subrange then extends to wavenumbers $k<0.1 k_{S}$, where the effects of the molecular diffusivity of salinity become important. Under energetic conditions, the high wavenumber limit of spectral resolution is often limited by the sampling volume of the SBE-07 ( $\sim 4 \mathrm{~mm})$, which is used in place of $0.1 k_{S}$ depending on which is smaller. With the inertial-convective and viscous-convective subranges thus defined, it is straightforward to estimate $\chi_{S}$ from the height of the spectra in conjunction with independent estimates of $\varepsilon$ from the ADVs (Fig. 8).

\section{Broadband acoustic backscattering system}

The acoustic system had four octave-bandwidth transducers spanning the frequency bands from 120 to $200 \mathrm{kHz}$ (LOW), 220 to $320 \mathrm{kHz}$ (MID), 350 to $470 \mathrm{kHz}$ (HL), and 450 to $600 \mathrm{kHz}(\mathrm{HH})$. The transducer half beamwidths at center frequency $\left(-3 \mathrm{~dB}\right.$ points) were $3^{\circ}$ for the LOW, $4.5^{\circ}$ for the MID, $5.5^{\circ}$ for the hearing level (HL), and $1.5^{\circ}$ for the $\mathrm{HH}$. The ping rates were $10 \mathrm{~Hz}$ on the MID frequency channel and $5 \mathrm{~Hz}$ on the LOW, HL, and HH frequency channels. All data collected in this study involved chirps of $500 \mu \mathrm{s} \mathrm{du}-$ ration. The system was calibrated using standard targets, following the procedures in Lavery et al. (2010a), based on protocols established by Foote and MacLennan (1984). Due to an error in the transmit power on the $\mathrm{HH}$ channel during calibration, an arbitrary $6 \mathrm{~dB}$ offset had to be applied to all the $\mathrm{HH}$ spectra to align them with the spectra for the other channel. The HH channel was consequently not used for any of the quantitative analysis. The broadband capabilities of the system are exploited through pulse-compression signal processing techniques (Turin, 1960; Chu and Stanton, 1998; Stanton and Chu, 2008; Stanton et al., 2010), which are based on matched filter processing and involve crosscorrelating the echo-voltage time-series with the transmitted 
(a) $\log _{10}(\varepsilon)$

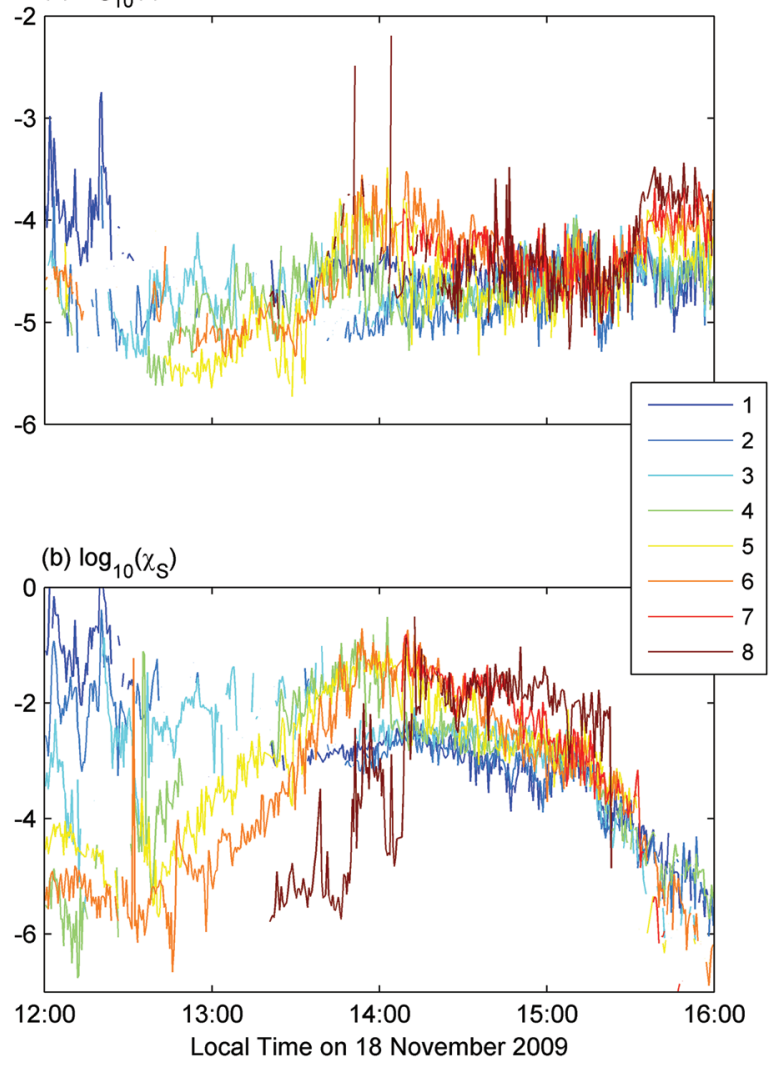

FIG. 8. Dissipation rate of (a) turbulent kinetic energy, $\varepsilon$, and (b) salinity variance, $\chi_{S}$, inferred from the eight in situ turbulence MAST sensors for the duration of the ebb tide during the anchor station on 18 November. The depth of the eight MAST sensors (shown in Fig. 13) varied slightly during the ebb tide as the angle of the MAST was adjusted due to the change of water depth with the ebbing tide. There is significantly higher variability in $\chi_{S}$ in than in $\varepsilon$, though both a relatively uniform during the mid-ebb (defined here as $13: 30$ to $15: 00)$.

signal voltage time-series. All acoustic images shown in this paper involve the envelope of the compressed-pulse output, $E_{\mathrm{CP}}$. This type of processing results in significantly increased temporal (and hence range) resolution, approximately equal to the inverse bandwidth $(1 / B)$, and increased signal-to-noise ratio (Stanton and Chu, 2008).

\section{E. Acoustic scattering spectra}

The focus of the analysis presented here is on the anchor station performed during an ebb tide on 18 November 2009. Acoustic spectra, defined as volume scattering $\left(S_{V}\right.$, defined below) versus wavenumber for all four channels, were calculated for all pings. The acoustic spectra were incoherently averaged (in linear space) over the number of pings contained in a $30 \mathrm{~s}$ period, a time period chosen to match the analysis of the MAST data. It is possible to average shorter acoustic records, approximately $1 \mathrm{~s}$, (corresponding to 5-10 pings) and still obtain good acoustic spectra, however, $30 \mathrm{~s}$ was the shortest time record that could be used with the MAST data and still obtain good spectral estimates (Geyer et al., 2008; Scully et al., 2011). 30 s corresponded to 300 pings for the MID frequency channel and 150 pings for the remaining channels, due to the different ping rates. However, 20- and 50-s temporal averaging periods were also used with broadly similar results. The depth range used to calculate the acoustic spectra was $20 \mathrm{~cm}$, chosen to be significantly smaller than the typical instabilities observed, which spanned peak-to-peak heights of 1-3 m. The smallest depth range that can be used is set by the inverse bandwidth of the signals used, which differs for the different channels, but is typically several $\mathrm{cm}$.

The volume sampled acoustically depends on the transducer bandwidth and beamwidth and changes with frequency and range (Fig. 9). Thus, for a particular ping sequence, the degree of overlap in the sampling volumes for the different transducers also changes with frequency and range. The LOW channel has a significantly larger sampling volume than the HH channel at all in-band frequencies, and a necessary assumption in comparing the scattering spectra across all four broadband transducers is that the scattering volume is known and that the source of volume scattering, such as, turbulent microstructure or suspended sediment, is homogeneous and fills the sampling volume. Furthermore, the degree to which consecutive pings are statistically independent depends on the natural evolution time of the scattering source in the volume (for example, the evolution time of salinity microstructure relative to the ping rate), as well as the fluid velocity, which results in advection of the scattering source through the acoustic scattering volume. The fluid velocity varied significantly with depth and time [Fig. 6(b)], with velocities as high as $1.35 \mathrm{~m} / \mathrm{s}$ observed in near surface waters, and negligibly small velocities encountered near the bottom. This combination of parameters resulted in a highly variable acoustic sampling volume and a large degree of variability in the statistical independence of consecutive pings.

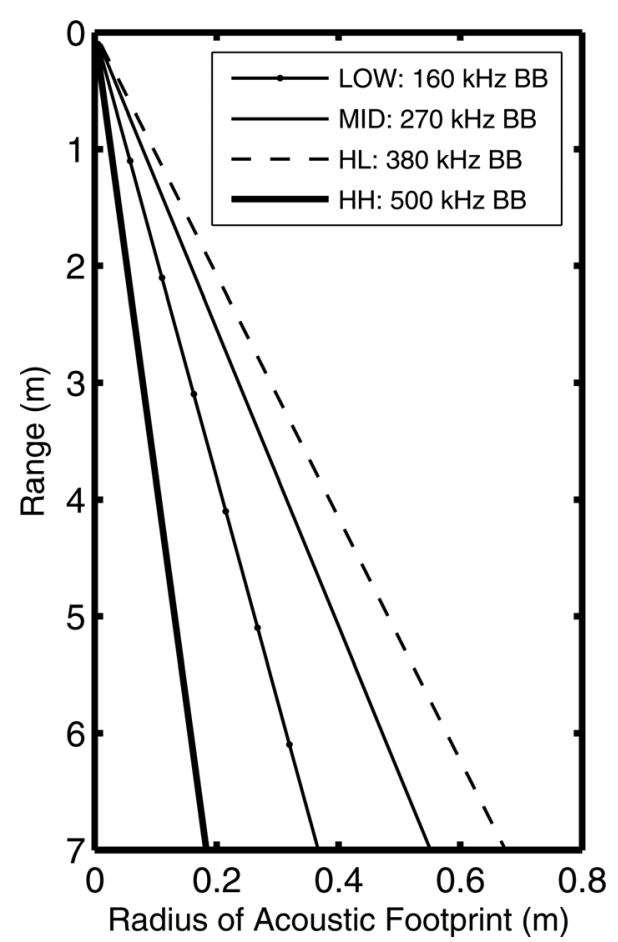

FIG. 9. Radius of the acoustic footprint as a function of range at the center frequencies of the four broadband transducers. Except for the HH channel, the acoustic footprints overlap. 
However, the relatively long temporal sampling window used ensured that most of this variability was averaged over.

\section{F. Ancillary measurements}

In addition to the measurements collected with the MAST and broadband backscattering system, continuous vertical profiles were conducted with a CTD sensor (Manufacturer RBR: $6 \mathrm{~Hz}$ sampling rate) deployed over the side (Fig. 5). The CTD was also equipped with a Seapoint optical backscattering sensor (OBS) which measured turbidity. Intermittent water samples and suspended sediment concentration measurements were also performed. Very fine sand dominated with median grain sizes of approximately $100 \mu \mathrm{m}$ (grain size analysis measurements courtesy of Jonathan Woodruff). Water samples were pumped from various depths along the MAST through 1-in. diameter tubing to determine the abundance and composition of small zooplankton and to allow the OBS to be calibrated. Subsequent analysis has shown that there were essentially no zooplankton present in the samples, and the measured abundances cannot account for even a small portion of the observed scattering.

\section{G. Parameters needed for predicting acoustic scattering spectra}

As stated earlier, in order to predict acoustic scattering from salinity microstructure it is necessary to estimate the parameters that make up $\Psi^{a c}$, namely, $A, B$, and $\partial T / \partial S$. These parameters are determined from the CTD profiles. In the CT River estuary there was a tight positive relationship between temperature and salinity (Fig. 10). Since $A$ and $B$ do not vary significantly (Fig. 11), a mean value of $\Psi^{a c}$ was used $\left(\Psi^{a c} \approx 3.49 \times 10^{-6} \mathrm{psu}^{-2}\right)$. A similar analysis was performed for $\Psi^{\mathrm{MAST}}$.

The spectra measured acoustically in the viscousconvective subrange follow the same power law as the

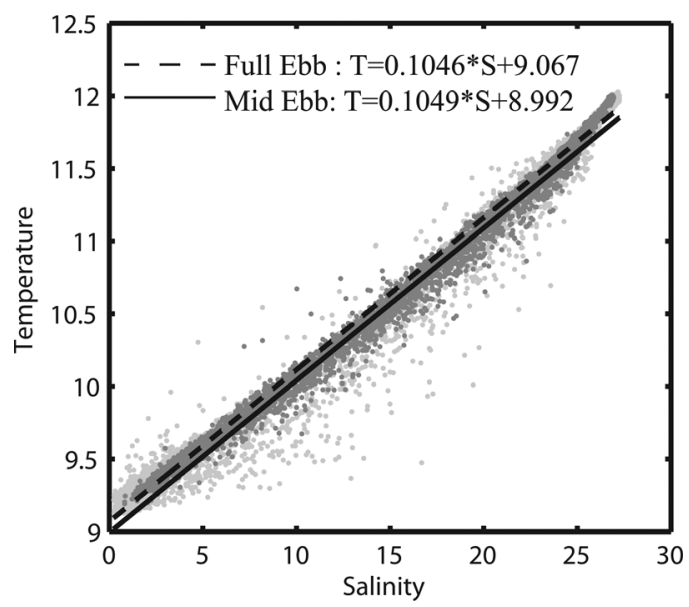

FIG. 10. Temperature-salinity plot for the anchor station on 18 November 2009, obtained from the continuous CTD profiles. The light gray points represent all measurements and the dark gray points represent the measurements obtained during the mid-ebb. The solid lack line represents the best least squares fit to the mid-ebb data and the dashed black line represents the best least squares fit to the data from the entire ebb. It can be seen that the slope changes very little.
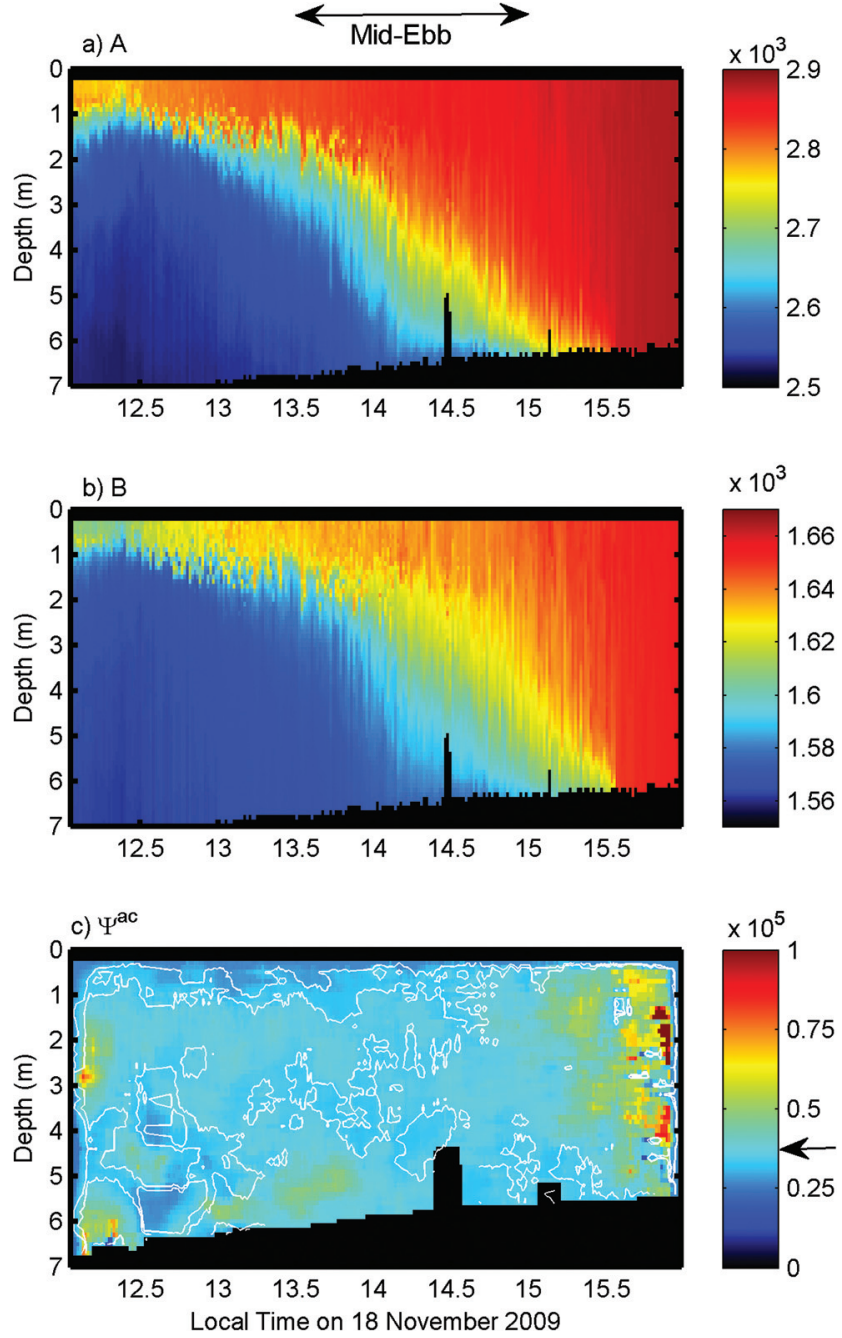

FIG. 11. The acoustic parameters (a) A, (b) B, and (c) $\Psi^{a c}$ throughout the ebb tide at the anchor station on 18 November 2009. Though these parameters vary throughout the ebb tide, it should be noted that the scale illustrates that these parameters only change a few percent, particularly during the mid-ebb portion of the tide. The black arrow in panel (c) indicates the value of $\Psi^{a c}$ used for the acoustic analyses.

spectra measured by the micro-conductivity sensors on the MAST in the viscous-convective subrange (Fig. 7), scaled by $2^{-3} \Psi^{a c}$, and, thus, can be almost directly compared. However, it should be noted that the MAST wavenumbers span both the inertial- and viscous-convective subranges, and, furthermore, do not overlap the wavenumbers spanned by the acoustic spectra (Fig. 6). Thus, it is necessary to perform a spectral fit to compare the spectral amplitudes.

\section{ACOUSTIC SPECTRAL CLASSIFICATION, QUANTIFICATION, AND COMPARISON TO MAST SPECTRA}

In order to use broadband acoustic scattering techniques as a quantitative remote sensing tool for quantifying stratified turbulence, it is important to be able to first classify the scattering sources. Two of the most common sources of scattering observed in the CT River estuary, in addition to stratified turbulence, were swim-bladdered fish and suspended sediments (Fig. 2). For the frequencies employed in this 
study, the scattering from suspended sediments, with grain diameters $<100 \mu \mathrm{m}$, is in the Rayleigh scattering regime with a clear $k^{4}$ scattering dependence (Medwin and Clay 1998), and the scattering from swim-bladdered fish is generally decreasing over this range of frequencies as a combined result of the resonance frequency occurring well below the lowest frequency available in this study and potential beampattern effects (Medwin and Clay, 1998). Thus, based on the clear differences in the wavenumber dependence of the observed scatterers in the CT River estuary, it is possible to perform spectral classification and quantification (Fig. 12).

Once the acoustic spectra had been calculated, a best fit power law was calculated in linear space. The power law exponent for the wavenumber, given by $N^{\alpha}$ (defined in Sec. II A), was used to determine the source of scattering [Fig. 13(a)]. Only acoustic spectra that had a power law broadly consistent with turbulence, for which $N^{\alpha}$ was chosen in the range $0<N^{\alpha}<5 / 3$ for this application, were compared to the MAST data. Because the acoustic wavenumbers were entirely

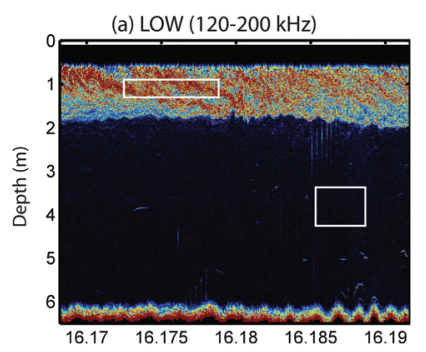

(c) $\mathrm{HH}(450-600 \mathrm{kHz})$

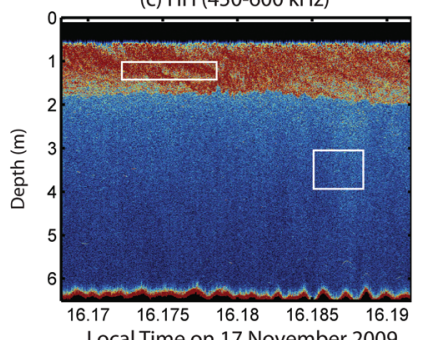

Local Time on 17 November 2009
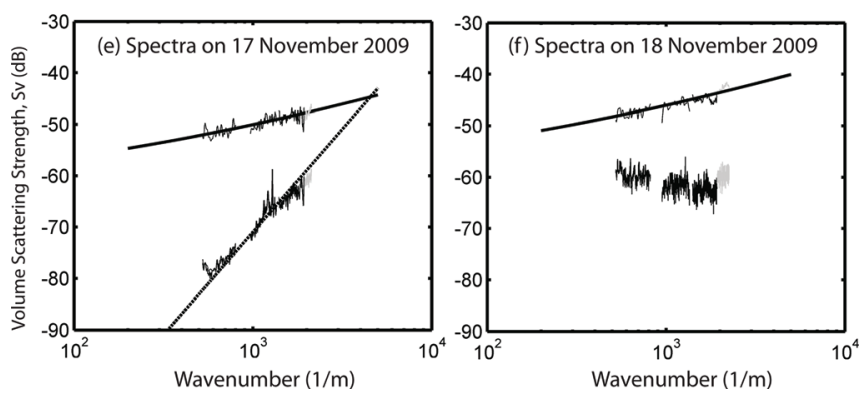

FIG. 12. Typical acoustic scattering spectra for different scatterers observed in the CT River estuary. Left hand panels: 17 November 2009. Right hand panels: 18 November 2008. Top panels: $\mathrm{E}_{\mathrm{CP}}$ for the LOW frequency band. Middle panels: $\mathrm{E}_{\mathrm{CP}}$ for the $\mathrm{HH}$ frequency band. Bottom panels: Acoustic scattering spectra, $S_{v}$ versus wavenumber, showing typical spectra for turbulence with a $k^{+1}$ spectrum, suspended sediment with a typical Rayleigh scattering regime wavenumber dependence of $k^{4}$, and swim-bladdered fish with a generally decreasing scattering spectrum. The solid lines show scattering predictions based on in situ turbulence parameters, and the dashed lines shows scattering predictions for suspended sediment using the mean measured fine sand grain size of $100 \mu \mathrm{m}$ and an arbitrarily adjusted concentration. The white boxes correspond to the pings and depth bins over which acoustic spectra were averaged to calculate the acoustic spectra.
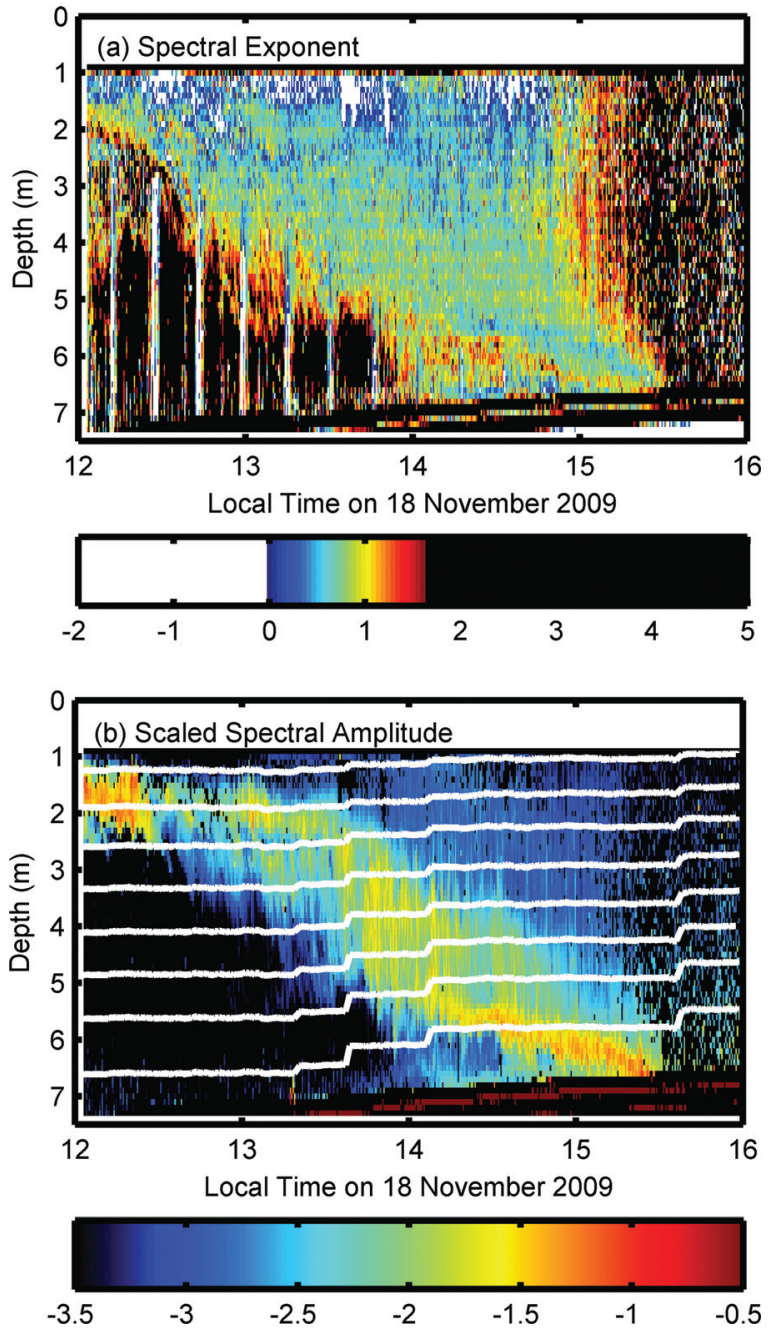

FIG. 13. (a) Best fit power law exponent determined from the acoustic spectra. (b) Scaled spectral levels for turbulent microstructure inferred acoustically, where only the spectra consistent with scattering from turbulence have been included. The white lines show the depths of the eight MAST sensors.

in the viscous-convective subrange (Figs. 2 and 7), the quantity that was compared to the MAST spectral levels [Eq. (6)] is $\left\langle\sigma_{V}^{\text {measured }}\right\rangle /\left(2^{-3} \Psi^{a c} k\right)=q \chi_{S}(\varepsilon / \nu)^{-1 / 2}=\phi_{S}^{v c}(k) k^{2}=S^{v c}(k)$ The acoustically inferred spectral levels throughout the ebb tide at the anchor station are shown in Fig. 13(b).

The spectral levels obtained from the MAST data are compared to the spectral levels inferred from the acoustic spectra in Fig. 14. These comparisons were performed at the eight different depths set by the MAST instrument brackets. The height of the MAST was adjusted throughout the deployment, as shown in Fig. 13(b), to adjust for the changing depth throughout the ebb tide. The acoustic and MAST spectral levels are relatively consistent during the mid-ebb, for times spanning $13.5-15 \mathrm{~h}$, when $\chi_{S}$ and $\varepsilon$ were particularly high and relatively stable throughout the water-column (Fig. 8). The agreement is best for the sensors spanning the mid-water-column (sensors 3-6). Late in the ebb (starting around $15.3 \mathrm{~h}$ ) the acoustic spectral heights are significantly larger than the spectral heights inferred from the MAST. Finally, based on the noise floor for the LOW frequency channel $(-82 \mathrm{~dB}$ at $120 \mathrm{kHz}$, corresponding to an acoustic 
wavenumber of $502 \mathrm{~m}^{-1}$ ), the smallest spectral level measured acoustically, assuming the scattering is entirely in the viscous-convective subrange, is given by $\left\langle\sigma_{V}^{v c}\right\rangle / 2^{-3}$ $\Psi^{a c} k=q \chi_{S}(\varepsilon / v)^{-1 / 2}=2.9 \times 10^{-5}\left(\mathrm{psu}^{2}\right)$. This minimum spectral level value can be substituted into Eq. (7) to determine the minimum values of $\varepsilon$ and $\chi_{S}$ measurable by the acoustic scattering system in a given stratification.

\section{COMPARISON OF ACOUSTIC AND MAST INFERENCES OF $\chi_{S}$ AND $\varepsilon$}

Though the primary goal of this work is to classify and quantify the acoustic spectral levels that are dominated by salinity microstructure, and compare them to measurements of in situ salinity spectra measured with the MAST (Fig. 14), it is possible to separately infer values of $\chi_{S}$ and $\varepsilon$ assuming a constant flux Richardson number and with knowledge of the salinity gradients [Eq. (7)], which can be obtained with a CTD and do not necessitate specialized instruments for measuring turbulent microstructure. A typical flux Richardson number for active turbulence under stratified conditions is given by $R_{f} \sim 0.15$ (Osborn, 1980), however, the spatial scales over which this general average is accurate, particularly in regions of either very active or very weak turbulence, represents an open research question (Ruddick et al., 1997; Smyth et al., 2001). Figure 15 compares the values of $\chi_{S}$ and

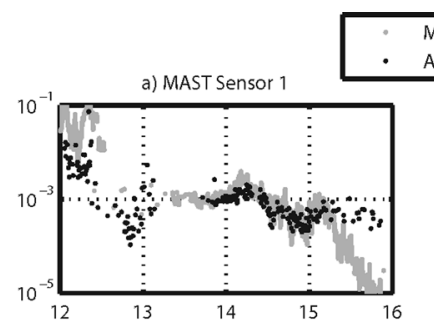

Acoustics
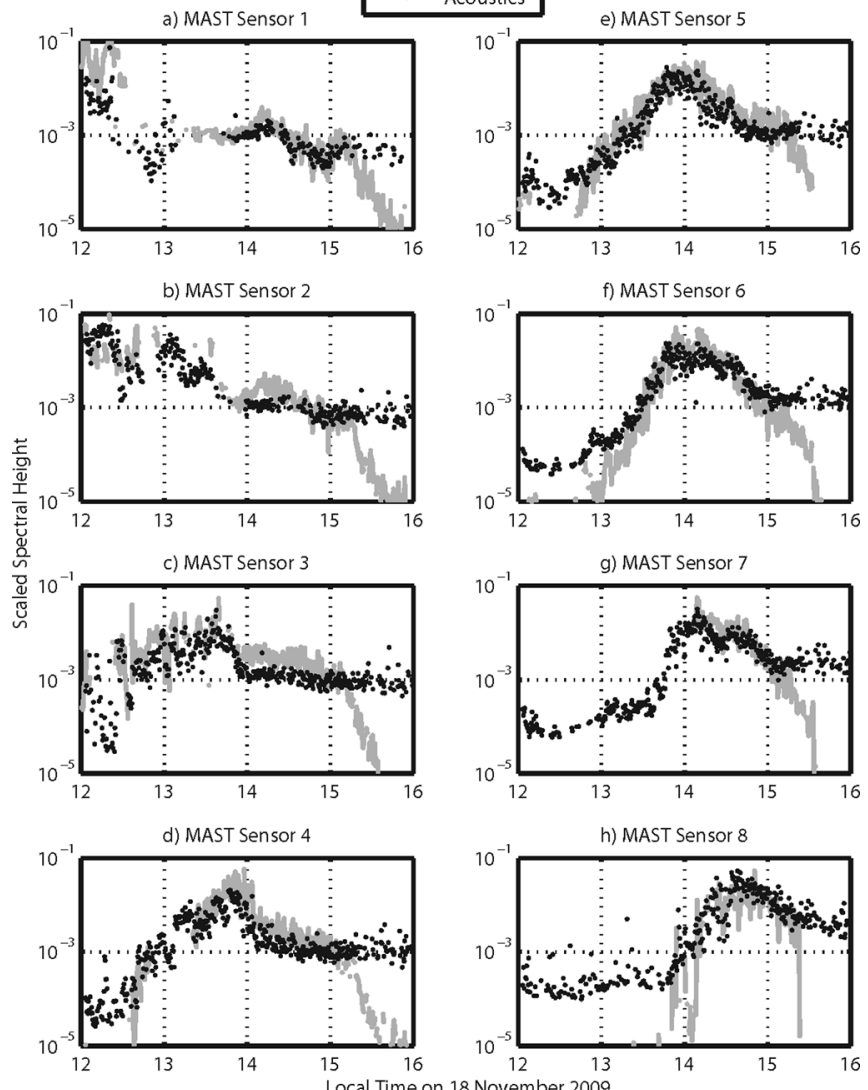

FIG. 14. Comparison of the scaled MAST spectral levels (gray lines) to the scaled spectral levels inferred acoustically (black lines) at the depths of the eight MAST sensors as a function of time during the ebb tide at the anchor station on 18 November 2009.

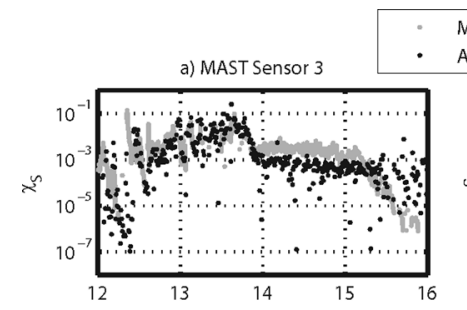

MAST
Acoustics
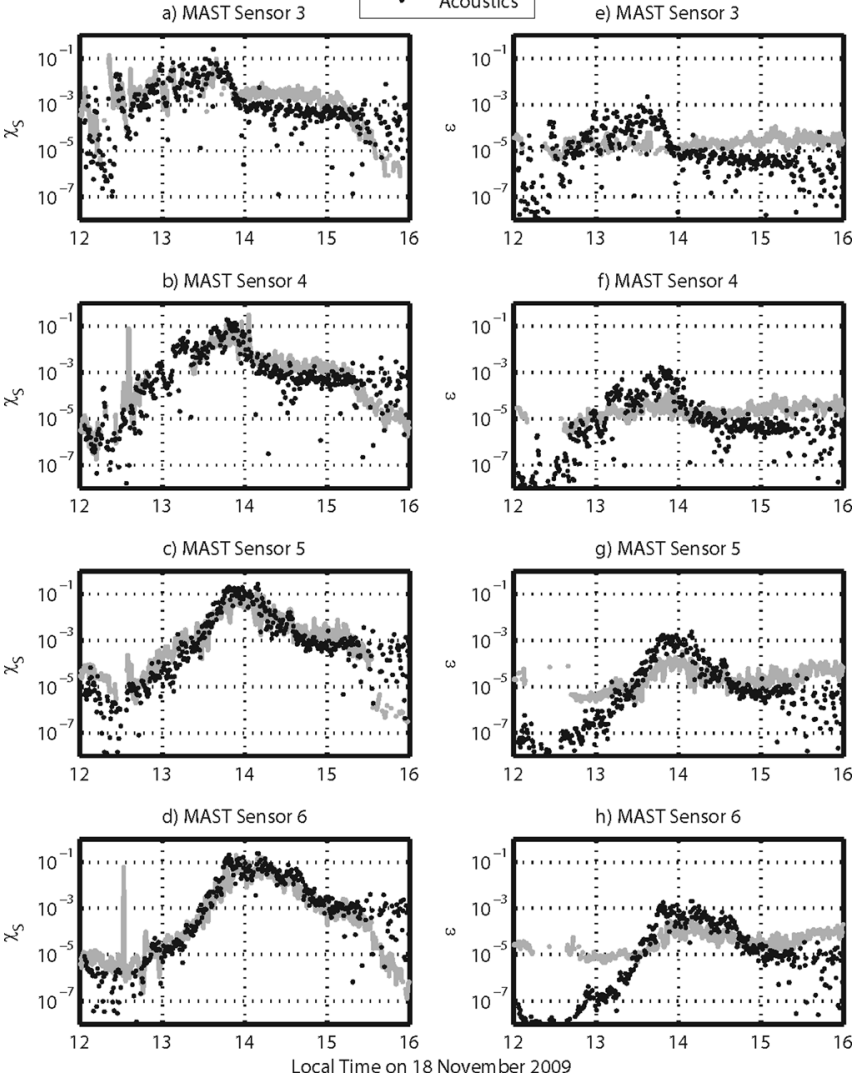

FIG. 15. Comparison of $\chi_{S}$ (left panels) and $\varepsilon$ (right panels), on a log scale, inferred from the in situ MAST (gray) and the broadband acoustic measurements (black) during the mid-ebb, for the MAST sensors located in the mid-water column, where the vertical shear and salinity gradient were almost linear increasing with depth during the mid-ebb. Salinity gradients measured by the MAST sensors and a constant flux Richardson value of $R_{f} \sim 15$ were used to infer $\chi_{S}$ and $\varepsilon$ from the acoustic data [Eq. (7)]. The agreement between the MAST and acoustic inferences of $\chi_{S}$ are better than those for $\varepsilon$ during the mid-ebb in part because of the greater sensitivity of the acoustic scattering to this parameter. The acoustic inferences of both $\chi_{S}$ and $\varepsilon$ during the late are ebb are significantly compromised due to a combination of very small salinity gradients [which appears in the denominator of Eq. (7)], which in turn results in lower scattering due to salinity microstructure, as well as higher suspended sediment loads, which lead to increased number of spectra that are incorrectly classified as due to salinity microstructure.

$\varepsilon$ inferred from the acoustic and MAST data with $R_{f}=0.15$. Only the sensors that span the mid-water-column are shown, as the agreement between the MAST and acoustic spectral heights was best for these sensors (Fig. 14). It can be seen that the acoustic inferences are consistent with the in situ MAST measurements during the mid-ebb, but there are significant discrepancies late in the ebb. The agreement between the MAST and acoustic inferences of $\chi_{S}$ are better than those for $\varepsilon$ during the mid-ebb, in part because of the greater sensitivity of the acoustic scattering to this parameter. This may be due in part to the limited range over which the ADVs can measure $\varepsilon$ and also the quadratic sensitivity of the acoustic estimate on $\partial S / \partial z$, which greatly increases the noise of the estimate when the salinity gradients are small during the late ebb. Furthermore, the acoustic inferences of both $\chi_{S}$ and $\varepsilon$ during the late ebb are significantly compromised due to a combination of very small salinity gradients [which appears in the denominator of Eq. (7)], which in turn results in lower 
scattering due to salinity microstructure, as well as higher suspended sediment loads, which lead to an increased number of spectra that are incorrectly classified as due to salinity microstructure.

\section{HIGH-RESOLUTION ACOUSTIC SPECTRA AND INFERENCES OF $\chi_{S}$}

It is possible to calculate acoustic spectra at higher temporal and spatial resolution than the $30-\mathrm{s}$ and $20-\mathrm{cm}$ averages that were used for comparison to the in situ MAST data during the anchor station. Figure 16(a) shows the acoustic spectral levels collected at high resolution (1-s and $2.5-\mathrm{cm}$ ) along a transect at the mouth of the CT River on 17 November 2009, corresponding to the inset in Fig. 1. This location was chosen because of the high scattering levels and clearly defined shear instabilities observable in the acoustic data but unresolved in the in situ MAST data. While the validity of the assumption of a constant flux Richardson number over these small spatial scales and in the presence of such active turbulence is an open research question, it is possible to perform the exercise of inferring values of $\chi_{S}$ at this very high spatial resolution [Fig. 16(b)]. From these inversions it can be seen that there are very high values of $\chi_{S}$ associated to the braids of the instabilities (Geyer et al., 2010), several orders of magnitude higher than in the cores of the instabilities. Furthermore, the spatial and temporal evolution of $\chi_{S}$ along

a) Scaled Spectral Amplitude

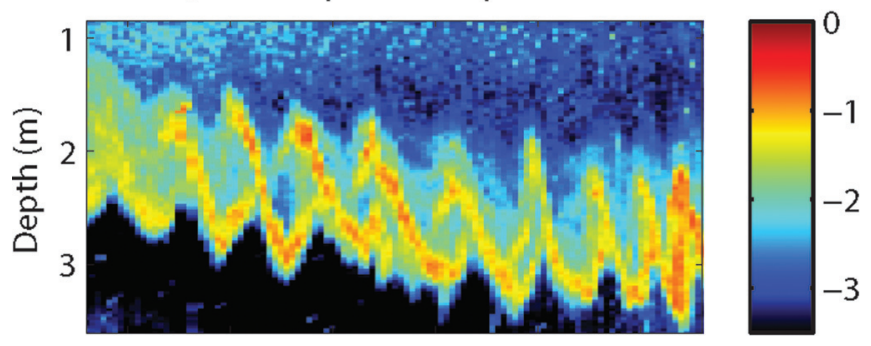

$\begin{array}{llllll}16.245 & 16.25 & 16.255 & 16.26 & 16.265 & 16.27\end{array}$

b) $\log _{10} \chi_{\mathrm{S}}$

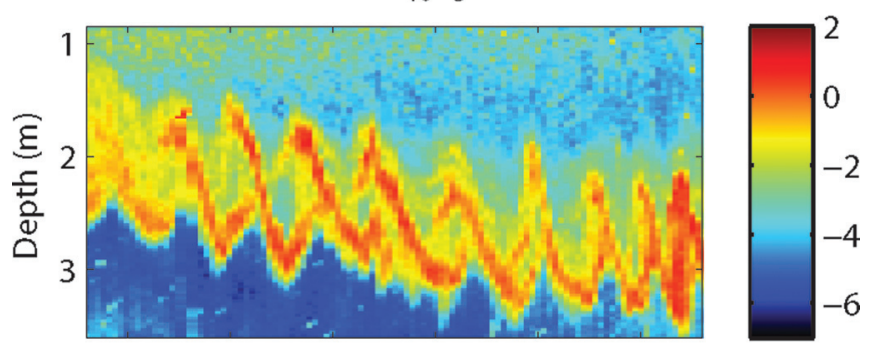

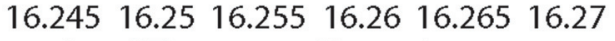

Local Time on 17 November 2009

FIG. 16. (a) Scaled spectral levels and (b) $\chi_{S}$, on a log scale, inferred from the broadband acoustic measurements at the mouth of the CT River on 17 November 2009, corresponding to the images shown in the inset of Fig. 1. The acoustic spectra were generated at high resolution, averaged over $1 \mathrm{~s}$ in tine and $5 \mathrm{~cm}$ vertically. It is not possible to compare these inferences with in situ MAST measurements due to the high resolution of the acoustic data. To infer $\chi_{S}$ from the acoustic spectral levels it was necessary to assume a constant mixing efficiency and to use a mean salinity gradient inferred from the MAST measurements. The broadband acoustic data predict high values of salinity dissipation in the braids of the shear instabilities. the braids can be seen, which could eventually lead to a better understanding of the transition to turbulence in strongly stratified environments.

\section{DISCUSSION}

The agreement between the acoustically inferred spectral levels for turbulent microstructure and the salinity spectral levels measured by the in situ micro-conductivity probes is best during the relatively homogeneous shear-induced mixing associated to the mid-ebb tide and when there were relatively few alternative sources of scattering present in the water-column (Fig. 14). During this time period the in situ measurements indicated that both $\chi_{S}$ and $\varepsilon$ were particularly high and stable throughout the water-column as compared with other times (Fig. 8). This results in high scattering levels, and thus better signal to noise ratios, and potentially smaller effects due to anisotropy (further discussion below). This agreement is particularly noteworthy in light of the fact that the physics based scattering model used to describe the scattering of sound from turbulent microstructure had no free parameters. It was necessary to have knowledge of the parameter $\Psi^{a c}$, however, this parameter does not vary considerably due to the tight T-S relationship in the CT River estuary and can be estimated relatively accurately from CTD profiles and does not require the need for sophisticated in situ microstructure measurements.

The spectral analysis of the acoustic data assumes a single scattering source, either turbulent microstructure, suspended sediments (or any small scatterer in the Rayleigh scattering regime), or fish. Though this is a great improvement over narrowband acoustic signals, it can still result in errors as it is possible that a combination of these scattering sources results in a spectrum that resembles turbulent microstructure. This was observed during the late ebb when suspended sediment loads increased. This kind of mixed scattering situation, in which the scattering was dominated at different frequencies by small zooplankton and turbulent temperature microstructure, was observed during the SW06 by Lavery et al. (2010). Furthermore, the spectral shape could be modified even in the absence of discrete sources of scattering, for example, if the temperature stratification were strong enough. As the molecular diffusivity of heat is two orders of magnitude larger than the molecular diffusivity of salt, the dissipation roll-off in the temperature spectrum, determined by the Batchelor wavenumber for temperature, $k_{B T}$, occurs at lower wave numbers than the dissipation rolloff in the salinity spectra, determined by the Batchelor wavenumber for salt, $k_{B S}$. As can be seen in Fig. 2, the diffusive roll-off for temperature occurs in the range of wavenumbers spanned by the broadband acoustic scattering system, potentially affecting the shape of the acoustic spectrum. However, as can be seen from Fig. 2, the temperature contribution is typically $20 \mathrm{~dB}$ lower than the salinity contribution, and thus it is not expected that the spectral shapes will be significantly modified by weak temperature stratification. Other alternative scattering sources that were not considered include 1) scattering from density and/or sound speed interfaces coherent over the scale of the acoustic scattering volume 
(analogous to reflection from a coherent fluid interface as reported by Lavery and Ross, 2007, for double-diffusive convection), and 2) the possibility that the strong stratification may have led to strong acoustic scattering even in the absence of active turbulence, due possibly to highly anisotropic small-scale density variations, possibly associated with decaying or "fossil" turbulence (Gibson, 1987).

The model employed in this study does not account for the potential impact of turbulence anisotropy. The influence of turbulence anisotropy on acoustic scattering is not generally very well understood, though Goodman (1990) suggested a scaling parameter that might account for the effects of anisotropy on acoustic scattering. Furthermore, measuring the degree of turbulence anisotropy at dissipation scales with in situ instrumentation is extremely challenging, resulting in a poor understanding of the conditions under which it is expected to contribute. It is generally thought that anisotropy at dissipation scales increases with decreasing values of $\varepsilon /\left(\nu N^{2}\right)$. For velocity gradient spectra, Gargett et al. (1984) suggest that an isotropic inertial subrange exists when $\varepsilon /\left(\nu N^{2}\right)>200$. Scalar gradient spectra are generally expected to be less isotropic (Sreenivasan, 1991), but Smyth and Moum (2000) demonstrate isotropy at dissipation scales when Cox numbers exceed $\mathrm{O}\left(10^{2}\right)$. The Cox number, $\mathrm{C}_{\mathrm{x}}$, is a non-dimensional quantity that is used commonly to characterize stratified turbulence, and is defined as the mean squared gradient of a scalar quantity mixed by turbulence, such as temperature or salinity, divided by the squared mean gradient. The excellent agreement between the acoustic and in situ data occurs during highly energetic conditions when $\varepsilon /\left(\nu N^{2}\right)>10^{3}$ and $\mathrm{C}_{\mathrm{x}} \gg 10^{3}$. Under these conditions anisotropy is unlikely to play a significant role in determining the scattering. However, under lower energy conditions, anisotropy is still expected to be present.

Additional bandwidth at the lower frequency end, for example spanning $30-120 \mathrm{kHz}$, would allow the inertialconvective subrange, as well as the lower portion of the viscous-convective subrange, to be probed acoustically. This would allow direct comparison of the MAST and acoustic spectra over the same wavenumber ranges, as the MAST measurements result in salinity spectra that span both the inertial-convective and viscous-convective subranges. This would circumvent the need to fit the acoustic data to a particular power law dependence. Due to limitations in the commercial availability of broadband transducers in this frequency band, the available broadband transducers have significantly broader beamwidths, and thus the resolution of individual shear instabilities might not be possible. However, with appropriate averaging, this approach would still be useful for inferring turbulence spectra remotely over a more extended wavenumber range. Additional bandwidth at the higher frequency range, might allow the salinity dissipation spectrum to be fully or partially resolved. If the salinity dissipation roll off could be resolved, then it might be possible to infer $\varepsilon$ from the location of the dissipation rolloff, which occurs at the Batchelor wavenumber for salinity, $k_{B S}$. However, this was not possible for the typical values of $\varepsilon$ encountered in this application as $k_{B S}$ occurred at wavenumbers beyond the available acoustic wavenumbers.
Furthermore, the very large values of $\varepsilon$ would require very high frequencies, order 5-10 MHz. At these frequencies, the range would be very restricted not only to due absorption, but also due to scattering by suspended sediments. It is also likely that suspended sediments will make up a larger fraction of the scattering at higher frequencies (the exact fractional contribution depending on the mean suspended sediment size), which might also obscure the roll-off in the salinity spectrum from the viscous-convective to the dissipation subrange. It should be noted that the in situ salinity spectra do not resolve the dissipation spectrum either, and thus the assumptions that are needed to estimate $\chi_{S}$ and $\varepsilon$ from the acoustic data are the same as the assumptions that are made for the in situ data.

\section{CONCLUSIONS}

The broadband acoustic backscattering measurements of highly salt-stratified turbulence in an estuary have resulted in remote inferences of turbulent salinity spectra. The acoustically inferred turbulent salinity spectra, which span wavenumbers in the viscous-convective subrange, agree well with salinity spectra determined with in situ turbulence measurements when averaged over similar temporal scales, as well as agreeing with theoretical acoustic scattering predictions. A key factor in determining the turbulent salinity spectra acoustically is the ability to capitalize on broadband signals to spectrally classify different water-column scatterers. Equally important were the high-stratification and energetic turbulence, resulting in high scattering signals. Inversions of the acoustically measured turbulent salinity spectra for relevant dissipation parameters revealed that the acoustic scattering is highly sensitive to the dissipation rate of salinity variance (as predicted by theory). Furthermore, when the same assumptions are made to infer dissipation rates from the acoustic and in situ turbulence measurements, the agreement between the dissipation parameters is remarkably good. This data set and analysis represents an important opportunity to better understand stratified turbulence and to advance the techniques for studying ocean mixing in general.

\section{ACKNOWLEDGMENTS}

The authors would like to thank the captain, Ken Houtler, and first mate, Ian Hanley, of the RV Tioga, for their assistance, experience, and willingness to work in the CT River, as well as Jay Sisson for construction and deployment of the instrumentation. The authors would also like to thank Jon Fincke and David Ralston for assistance in collecting the data. This work was supported by NSF grant OCE0824871, ONR grant N00014-0810495, and WHOI internal funds.

Batchelor, G. K. (1959). "Small-scale variation of convective quantities like temperature in a turbulent fluid," J. Fluid Mech. 5, 113-133.

Chu, D., and Stanton, T. K. (1998). "Application of pulse compression techniques to broadband acoustic scattering by live individual zooplankton," J. Acoust. Soc. Am. 104, 39-55. 
Cummins, P. F., Armi, L., and Vagle, S. (2006). "Upstream internal hydraulic jumps," J. Phys. Oceangr. 36(5), 753-769.

Dillon, T. M., and Caldwell, D. R. (1980). "The Batchelor spectrum and dissipation in the upper ocean," J. Geophys. Res. 85, 1910-1916, doi:10.1029/JC085iC04p01910.

Farmer, D. M., and Armi, L. (1999). "The generation and trapping of internal solitary waves over topography," Science 283, 188-190.

Farmer, D. M., and Smith, J. D. (1979). "Tidal interaction of stratified flow with a sill in Knight inlet," Deep-Sea Res., Part A 27, 239-254.

Foote, K. G., Atkins, P. R., Francis, D. T. I., and Knutsen, T. (2005). "Measuring echo spectra of marine organisms over a wide bandwidth," in Proceedings of the International Conference on Underwater Acoustic Measurements: Technologies and Results, Heraklion, Crete, Greece, 28 June-1 July 2005, edited by J. S. Papadakis, and L. Bjørnø (FORTHIACM, Heraklion, Crete), pp. 501-508.

Foote, K. G., and MacLennan, D. N. (1984). "Comparison of copper and tungsten carbide calibration spheres," J. Acoust. Soc. Am. 75, 612-616.

Gargett, A. E., T. R. Osborn, and Nasmyth, P. W. (1984). "Local isotropy and the decay of turbulence in a stratified fluid," J. Fluid Mech. 144, 231-280.

Geyer, W. R., Lavery, A. C., Scully, M. E., and Trowbridge, J. H. (2010). "Mixing by shear instability at high Reynolds number," Geophys. Res. Lett. 37, L22607, doi:10.1029/2010GL045272.

Geyer, W. R., Scully, M. E., and Ralston, D. K. (2008). "Quantifying vertical mixing in estuaries,” Environ. Fluid Mech. 8, 495-509.

Gibson, C. H. (1987). "Fossil turbulence and intermittency in sampling oceanic mixing processes,” J. Geophys. Res. 92, 5383-5404, doi:10.1029/ JC092iC05p05383.

Goodman, L. (1990). "Acoustic scattering from ocean microstructure," J. Geophys. Res. 95, 11557-11573.

Goodman, L., and Sastre-Conrdova, M. M. (2011). "On observing acoustic scattering from salinity turbulence," J. Acoust. Soc. Am. 130, 707-715.

Grant, W. D., Williams III, A. J., and Glenn, S. M. (1984). "Bottom stress estimates and their prediction on the Northern California continental shelf during CODE-1: The importance of wave-current interaction," J. Phys. Oceanogr. 14, 506-527.

Haury, T. R., Briscoe, M. G., and Orr, M. H. (1979). "Tidally generated internal wave packets in Massachusetts Bay," Nature 278(5702), 312-317.

Hay, A. E. (1983). "On the remote acoustic detection of suspended sediment at long wavelengths," J. Geophys. Res. 88, 7525-7542, doi:10.1029/ JC088iC12p07525.

Kraichnan, R. H. (1953). "The scattering of sound in a turbulent medium," J. Acoust. Soc. Am. 25, 1096-1104.

Lavery, A. C., Chu, D., and Moum, J. N. (2010a). "Measurements of acoustic scattering from zooplankton and oceanic microstructure using a broadband echosounder," ICES J. Mar. Sci. 67, 379-394.

Lavery, A. C., Chu, D., and Moum, J. N. (2010b). "Observations of broadband acoustic backscattering from nonlinear internal waves: assessing the contribution from microstructure," IEEE J. Ocean. Eng. 34, 695-709.

Lavery, A. C., and Ross, T. (2007). "Acoustic scattering from doublediffusive microstructure," J. Acoust. Soc. Am. 122, 1449-1462.

Lavery, A. C., Schmitt, R. W., and Stanton, T. K. (2003). "High-frequency acoustic scattering from turbulent oceanic microstructure: The importance of density fluctuations," J. Acoust. Soc. Am. 114, 2685-2697.

Lynch, J. F., Gross, T. F., Brumley, B. H., and Filyo, R. A. (1991). "Sediment concentration profiling in HEBBLE using a 1-MHz acoustic backscatter system," Mar. Geol. 99, 361-385.

Medwin, H. (1977). "In situ acoustic measurements of microbubbles at sea," J. Geophys. Res. 82, 971-976, doi:10.1029/JC082i006p00971.

Medwin, H., and Clay, C. S. (1998). Fundamentals of Acoustical Oceanography (Academic, Boston, MA), Chap. 9.

Moum, J. N., Farmer, D. M., Smyth, W. D., Armi, L., and Vagle, S. (2003). "Structure and generation of turbulence at interfaces strained by internal solitary waves propagation shoreward over the continental shelf," J. Phys. Oceanogr. 33, 2093-2122.

Nash, J. D., and Moum, J. N. (1999). "Estimating salinity variance dissipation rate from conductivity microstructure measurements," J. Atmos. Ocean. Technol. 16, 263-274.

Nash, J. D., and Moum, J. N. (2002). "Microstructure estimates of turbulent salinity flux and the dissipation spectrum of salinity," J. Phys. Oceanogr. 32, 2312-2330
Oakey, N. S. (1982). "Determination of the rate of dissipation of turbulent energy from simultaneous temperature and shear microstructure measurements," J. Phys. Oceanogr. 12, 256-271.

Orr, M. H., Haury, L. R., Wiebe, P. H., and Briscoe, M. G. (2000). "Backscatter of high-frequency $(200 \mathrm{kHz})$ acoustic wave fields from ocean turbulence," J. Acoust. Soc. Am. 108, 1595-1601.

Osborn, T. R. (1980). "Estimates of the Local Rate of Vertical Diffusion from Dissipation Measurements," J. Phys. Oceanogr. 10, 83-89.

Proni, J. R., and Apel, J. R. (1975). "On the use of high-frequency acoustics for the study of internal waves and microstructure," J. Geophys. Res. 80, 1147-1151, doi:10.1029/JC080i009p01147..

Ross, T., Gaboury, I., and Lueck, R. (2007). "Simultaneous acoustic observations of turbulence and zooplankton in the ocean," Deep-Sea Res. I 54, $143-153$.

Ross, T., Garrett, C., and Lueck, R. (2004). "On the turbulent co-spectrum of two scalars and its effect on acoustic scattering from oceanic turbulence," J. Fluid Mech. 514, 107-119.

Ross, T., and Lueck, R. (2003). "Sound scattering from oceanic turbulence," J. Geophys. Res. Lett. 30, 1343-1346, doi:10.1029/2002GL016733.

Ruddick, B., Walsh, D., and Oakey, N. (1997). "Variations in apparent mixing efficiency in the North Atlantic central water," J. Phys. Oceanogr. 27, 2589-2605.

Sandstrom, H., Elliott, J. A., and Cochrane, N. A. (1989). "Observing groups of solitary internal waves and turbulence with BATFISH and echosounder," J. Phys. Oceanogr. 19(7), 987-997.

Scully, M. E., Geyer, W. R., and Lerczak, J. A. (2009). "The influence of lateral advection on the residual estuarine circulation: a numerical modeling study of the Hudson River estuary," J. Phys. Oceanogr. 39, 107-124.

Scully, M. E., Geyer, W. R., and Trowbridge, J. H. (2011). "The influence of stratification and non-local turbulent production on estuarine turbulence: An assessment of turbulence closure with field observations," J. Phys. Oceanogr. 41, 166-185.

Seim, H. E. (1999). "Acoustic backscatter from salinity microstructure," J. Atmos. Ocean. Technol. 16, 1491-1498.

Seim, H. E., Gregg, M. C., and Miyamoto, R. T. (1995). "Acoustic backscatter from turbulent microstructure," J. Atmos. Ocean. Technol. 12, $367-380$.

Seuront, L., Schmitt, F., and Lagadeuc, Y. (2001). "Turbulence intermittency, small-scale phytoplankton patchiness and encounter rates in plankton: Where do we go from here?," Deep-Sea Res. I 48(5), 1199-1215.

Shaw, W. J., Trowbridge, J. H., and Williams III, A. J. (2001). "Budgets of turbulent kinetic energy and scalar variance in the continental shelf bottom boundary layer," J. Geophys. Res. 106(C5), 9551-9564, doi:10.1029/ $2000 J C 000240$.

Smyth, W. D., and Moum, J. N. (2000). "Anisotropy of turbulence in stably stratified mixing layers," Phys. Fluids 12, 1343-1362, available at http:// dx.doi.org/10.1063/1.870386.

Smyth, W. D., Moum, J. N., and Caldwell, D. R. (2001). "The Efficiency of Mixing in Turbulent Patches: Inferences from Direct Simulations and Microstructure Observations," J. Phys. Oceanogr. 31, 1969-1992.

Sreenivasan, K. R. (1991). "On local isotropy of passive scalars in turbulent shear flows," Proc. R. Soc. London, Ser. A 434, 165.

Sreenivasan, K. R. (1995). "On the universality of the Kolmogorov constant," Phys. Fluids 7, 2778-2784.

Stanton, T. K., and Chu, D. (2008). "Calibration of broadband active acoustic systems using a single standard spherical target," J. Acoust. Soc. Am. 124, 128-136.

Stanton, T. K., Chu, D., Jech, J. M., and Irish, J. D. (2010). "New broadband methods for resonance classification and high-resolution imagery of fish with swim-bladders using a modified commercial broadband echosounder," ICES J. Mar. Sci. 67, 365-378.

Tang, D. J., Moum, J. N., Lynch, J., Abbot, P., Chapman, R., Dahl, P. H., Duda, T. F., Gawarkiewicz, G., Glenn, S., Goff, J. A., Graber, H., Kemp, J., Mafferi, A., Nash, J. D., and Newhall, A. (2007). "Shallow Water'06: A joint acoustic propagation/nonlinear internal wave physics experiment," Oceanography 20, 156-158.

Tennekes, H., and Lumley, J. L. (1972). A First Course in Turbulence (The MIT Press, Cambridge, MA), 320 pp.

Thorne, P. D., Vincent, C. E., Hardcastle, P. J., Rehman, S., and Pearson, N. (1991). "Measuring suspended sediment concentrations using acoustic backscatter devices," Mar. Geol. 98, 7-16.

Thorpe, S. A., and Brubaker, J. M. (1983). "Observation of sound reflection by temperature microstructure,” Limnol. Oceanogr. 28, 601-613. 
Thorpe, S. A., Cure, M. S., and Graham, A. (1994). "Sonar observations of Langmuir circulation and estimation of dispersion of floating particles," J. Atmos. Ocean. Technol. 11, 1273-1294.

Trevorrow, M. V. (1998). "Observations of internal solitary waves near the Oregon coast with an inverted echo sounder," J. Geophys. Res. 103, 7671-7680, doi:10.1029/98JC00101.

Turin, G. L. (1960). "An introduction to matched filters," IRE Trans. Inf.Theory IT-6, 311-329.

Vagle, S., and Farmer, D. M. (1994). "The measurement of bubble-size distributions by acoustical backscatter," J. Atmos. Ocean. Technol. 9, 630-644.
Warren, J. D., Stanton, T. K., Wiebe, P. H., and Seim, H. E. (2003). "Inference of biological and physical parameters in an internal wave using multiple-frequency, acoustic-scattering data," ICES J. Mar. Sci. 60, 1033-1046.

Washburn, L., Duda, T. F., and Jacobs, D. C. (1996). "Interpreting conductivity microstructure: Estimating the temperature variance dissipation rate," J. Atmos. Ocean. Technol. 13, 1166-1188.

Young, R. A., Merrill, J. T., Clarke, T. L., and Proni, J. R. (1982). "Acoustic profiling of suspended sediments in the marine bottom boundary layer," Geophys. Res. Lett. 9, 175-178, doi:10.1029/GL009i003p00175. 